\title{
A 3-phase model for the numerical analysis of semi- crystalline polymer films in finite elastoplastic strains
}

\author{
Philippe Le Grognec ${ }^{a,}{ }^{,}$, Salim Chaki ${ }^{a}$, Fanfei Zeng ${ }^{a}$, Mélanie Nottez ${ }^{a}$ \\ ${ }^{a}$ Mines Douai
}

Polymers and Composites Technology \& Mechanical Engineering Department

941 rue Charles Bourseul - CS 10838, 59508 Douai Cedex, France

\begin{abstract}
In this paper, the mechanical behavior of semi-crystalline polymer films in finite elastoplastic strains is investigated. A 3-phase constitutive model has been specially developed in a previous paper and validated for various materials in both uniaxial and biaxial uniform hot drawing. In the present study, the numerical implementation of this 3-phase model in a finite element software is outlined in the perspective of using this model in more general non-uniform cases of complex geometries and/or loadings. In the present case, only polyethylene (PE) films at room temperature are considered. First, uniaxial tensile experimental tests are performed so as to calibrate the model parameters. Then, for validation purposes, two series of experimental tests are conducted on tensile specimens with central holes and Double Edge Notched Tensile (DENT) specimens. During these tests, Digital Image Correlation is used to analyze the strain (or displacement) field history during loading. Finally, numerical computations are performed with the help of the finite element software including the 3phase model previously implemented (cohesive elements are also needed for the simulation of the crack propagation in DENT specimens). In both cases, the comparison between the experimental and numerical force-displacement curves, together with the comparisons between the experimental and numerical strain fields at different times, give very satisfactory results.
\end{abstract}

Keywords: Semi-crystalline polymers, Finite elastoplasticity, Numerical implementation, Cohesive elements, Digital image correlation.

* Corresponding author.

Email addresses: philippe.le.grognec@mines-douai.fr (Philippe Le Grognec), salim.chaki@mines-douai.fr (Salim Chaki), fanfei.zeng@mines-douai.fr (Fanfei Zeng), melanie.nottez@mines-douai.fr (Mélanie Nottez). 


\section{Introduction}

The demand of multi-functional materials has significantly increased over the past years. In the particular field of food packaging, multi-layer blown films offer a great potentiality since they can simultaneously address many performance criteria (in terms of mechanical, optical and gas-barrier properties, for example) in combination with an easy processability. Among others, the combination of polyethylene (PE) and ethylene vinyl alcohol (EVOH) has recently shown a particular interest. Indeed, PE is an excellent barrier to moisture and provides a good mechanical strength, whereas $\mathrm{EVOH}$ presents a very good barrier effect to oxygen, aromatics and oils [1].

Modeling the mechanical behavior of such multi-layer films is an important objective for industrial applications. It has been clear for a long time that standard elastoplastic formulations are not suitable for describing the behavior of semi-crystalline polymers, due to their complex morphology. Over the past decades, many constitutive models were thus proposed in the literature to simulate the mechanical behavior of semi-crystalline polymers, among which one can identify phenomenological and physical models. Dealing with the physically-based approaches, many network models were first suggested (among which the classical 8-chain model), emphasizing the rubber-like properties of the amorphous phase of semi-crystalline polymers [2,3]. Then, based on these fundamental ideas, numerous formulations were proposed with the aim of simulating the (thermo-)mechanical response of amorphous or semi-crystalline polymers [4-6]. In particular, many models were developed as a generalization of the 8-chain model for predicting the large strains, time- and/or temperature-dependent elastoplastic response of ultra-high molecular weight polyethylene, as reviewed in [7]. Lee et al. [8] developed a specific model in order to study the large plastic deformation and texture evolution in isotropic high-density polyethylene. Dupaix and Boyce [9] developed a constitutive model in order to capture the rate-dependent stress-strain behavior of poly(ethylene terephthalate)-glycol at and above the glass transition temperature. Richeton et al. [10] modified the 8-chain model so as to simulate the behavior of poly(methyl methacrylate) and polycarbonate, taking into account the influence of relaxation in polymer chains.

In many cases, two models are used in parallel to represent the combined effect of amorphous chains and crystalline skeleton. Following this idea, Gueguen et al. [11] investigated the elasto(visco)-plastic behavior of semi-crystalline polymers using a micromechanically-based formulation, considering two phases (crystalline and amorphous) with independent yield processes. Ayoub et al. [12] studied the mechanical stress-strain response of polyethylene under finite strains using a twophase representation of the microstructure. The constitutive response of both crystalline and amorphous phases was experimentally determined and the model was validated over a wide range of crystallinities. In a slightly different way, the evolution of void fraction and shape during uniaxial stretching of semi-crystalline and amorphous polymers was analyzed by Ponçot et al. [13], in order to derive their true intrinsic mechanical behavior. Two multi-phase models, which also take into account the degree of crystallinity, were developed and implemented in a finite element software by Regrain et al. [14] in order to investigate the creep behavior of semi-crystalline polymers. In view of biomedical applications, Khan et al. [15] characterized the mechanical properties of a ultra-high molecular weight polyethylene and used a viscoplasticity theory based on overstress for creep modeling purposes. 
The yield behavior of semi-crystalline polymers was particularly studied by Farrokh and Khan [16]. Based on experimental predictions, they proposed an empirical equation in order to express the strain rate sensitivity of the yield value. In Rozanski and Galeski [17], the influence of swelling of the amorphous phase on the yield stress of semi-crystalline polymers was studied experimentally. The yield behavior of high-density polyethylene under multiaxial loading conditions was examined by Hachour et al. [18]. The relevance of several classical yield criteria, involving both the deviatoric and hydrostatic stresses, was verified for various stress states.

Lastly, let us cite Srivastava et al. [19] who developed a large-deformation, thermomechanically-coupled elastic-viscoplastic theory so as to investigate the mechanical behavior of polymers at temperatures below and above the glass transition. Likewise, Uchida et Tada [20] analyzed the deformation mechanisms of semi-crystalline polymers at microscopic, mesoscopic and macroscopic scales and built a computational procedure relating each scale to the others with a view to simulating iteratively the global mechanical response. Except the last ones, most of the previous models have only been used and thus validated in the context of uniaxial drawing. As an example, Maurel-Pantel et al. [21] developed an original and promising thermo-mechanical elastic-viscoplastic large deformation constitutive model in a 3D framework, based on a previous 1D visco-hyperelastic model initially developed for amorphous rubbery polymers, but up to now, it was not implemented in a finite element code.

Recently, a special (rate-independent) elastoplastic model was introduced by Zeng et al. [22], with the objective of catching both uniaxial and biaxial behaviors of semi-crystalline polymers (at low strain rates) in a unified way. The main features of this model are: (i) a 3-phase description of the morphology and hence of the constitutive behavior; (ii) a modified von Mises criterion based on a multi-axial factor. This simple model, involving just a few parameters to calibrate, has already shown its ability to reproduce both uniaxial and equi-biaxial behaviors (with their noteworthy double yielding points) of various polymers under different temperatures. The theoretical predictions have found to be in very good accordance with experimental results up to very large irreversible strains.

In practice, the mechanical response of polymer films is not necessarily uniform. The presence of initial defects (such as holes or cracks) gives rise to stress concentrations. Even in the case of an unnotched sample, strain localization effects are likely to occur, transient ones for high strain rates [23] but also permanent ones for low strain rates [24-26]. Therefore, one has to focus not only on the global force-displacement curve (or local stress-strain curve) but also on the displacement (or strain) field throughout the sample in hand. From an experimental point of view, the measurement of displacement fields, even at moderately high strain rates, is feasible today with modern high-speed camera systems [23]. The widespread use of Digital Image Correlation (DIC), and its coupling with the technology of high-speed cameras, may provide the desired displacement fields [25]. The basic concept of DIC was proposed in the eighties by Sutton et al. $[27,28]$ and recent researches using this technique have been performed, regarding the evaluation of local displacements in polymeric materials $[18,29,30]$. From a numerical point of view, the use of a finite element software allows one to predict the general non-uniform response of polymer films including possible defects.

In light of this, the present paper aims to analyze the uniaxial response of various unnotched and notched specimens of a polyethylene blown film. More specifically, three kinds of samples will be used: unnotched rectangular specimens, rectangular specimens with a hole at the center, and 
Double Edge Notched Tensile (DENT) specimens. The unnotched specimens (with a uniform response) will be used for the calibration of the 3-phase model. Then, the case of open-hole specimens, which is experimentally and geometrically easy to handle, allows one to deal with multiaxial stress/strain states and high stress concentrations, and it represents an intermediate stage between the simple case of unnotched specimens and the more complex analysis of DENT specimens, where stresses become singular near the crack tips. The problem of crack propagation in DENT specimens will be studied lastly, so as to illustrate one of the major failure modes of such polymer films.

On one hand, experimental tensile tests will be performed and post-processed with the help of 2D Digital Image Correlation. On the other hand, the 3-phase model developed by some of the authors [22] will be implemented in a commercial finite element software. Numerical computations will be performed for all kinds of samples (involving not only the 3-phase model but also specific cohesive elements at the expected place of the crack evolution in the case of DENT specimens) and the results will be confronted to experimental curves and fields, in order to evaluate the efficiency of the present model in such extreme multi-axial conditions.

\section{Experimental data}

\subsection{Materials}

The materials considered in this study are a low density polyethylene (LDPE), grade FE 8000, from Total Petrochemicals (France), and a metallocene ethylene-hexene copolymer (m-LDPE), grade Exceed 2018CA, purchased by ExxonMobil Chemicals (USA). The main characteristics of the raw materials are reported in Table 1.

Table 1. Material properties

\begin{tabular}{|c|c|c|c|c|c|}
\hline Materials & Supplier & Reference & $\begin{array}{c}\text { Melt flow } \\
\text { index } \\
(\mathrm{g} / 10 \mathrm{~min})\end{array}$ & $\begin{array}{c}\text { Density } \\
\left(\mathrm{g} / \mathrm{cm}^{3}\right)\end{array}$ & $\begin{array}{c}\text { Melting } \\
\text { temperature } \\
\left({ }^{\circ} \mathrm{C}\right)\end{array}$ \\
\hline LDPE & $\begin{array}{c}\text { TOTAL } \\
\text { PETROCHEMICALS } \\
\text { (France) }\end{array}$ & FE 8000 & 0.8 & 0.924 & 111 \\
\hline m-LDPE & $\begin{array}{c}\text { EXXONMOBIL } \\
\text { CHEMICALS (USA) }\end{array}$ & $\begin{array}{c}\text { EXceed } \\
\text { 2018CA }\end{array}$ & 2.0 & 0.918 & 118 \\
\hline
\end{tabular}

\subsection{Manufacturing process}

A three-layer film (LDPE / LDPE / m-LDPE) was manufactured at $180^{\circ} \mathrm{C}$ on a three-line coextrusion device (Collin) equipped with a blow die of inner diameter $50 \mathrm{~mm}$ and gap width $0.8 \mathrm{~mm}$ (see the main characteristics in Table 2). The blow-up ratio was fixed at 3.4 and the maximum deviation of the film thickness about the average value was $\pm 10 \mu \mathrm{m}$. 
Table 2. Characteristics of the co-extrusion line and manufacturing conditions

\begin{tabular}{|c|c|c|c|c|}
\hline \multirow{2}{*}{ Layer } & \multicolumn{2}{|c|}{ Screw } & \multicolumn{2}{c|}{ Extrusion conditions } \\
\cline { 2 - 5 } & Diameter $D(\mathrm{~mm})$ & Length & Thickness $(\mu \mathrm{m})$ & $\begin{array}{c}\text { Extrusion } \\
\text { temperature }\left({ }^{\circ} \mathrm{C}\right)\end{array}$ \\
\hline Internal (m-LDPE) & 25 & $25 \mathrm{D}$ & 20 & 180 \\
\hline Central (LDPE) & 30 & $30 \mathrm{D}$ & 40 & 180 \\
\hline External (LDPE) & 25 & $25 \mathrm{D}$ & 20 & 180 \\
\hline
\end{tabular}

\subsection{Mechanical testing}

Experimental tensile tests have been performed on a Lloyd LR 50K material-testing machine mounted with a $100 \mathrm{~N}$-force cell at a constant crosshead speed of $50 \mathrm{~mm} \cdot \mathrm{min}^{-1}$, at room moisture and $25^{\circ} \mathrm{C}$, according to the ISO 527.3 standard. Three types of specimen were tested: unnotched, open-hole and Double Edge Notched Tensile (DENT) ones (two initial crack lengths were considered in the last case). Four samples were tested in each case (to check reproducibility) with in-plane dimensions reported in Figure 1 and a thickness varying from 61 to $88 \mu \mathrm{m}$.

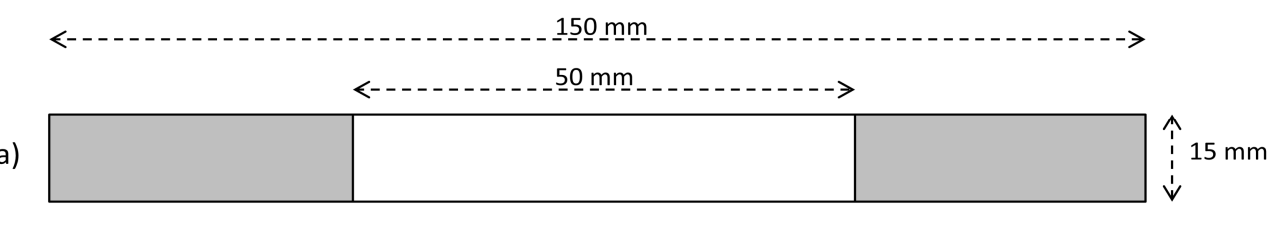

(b)
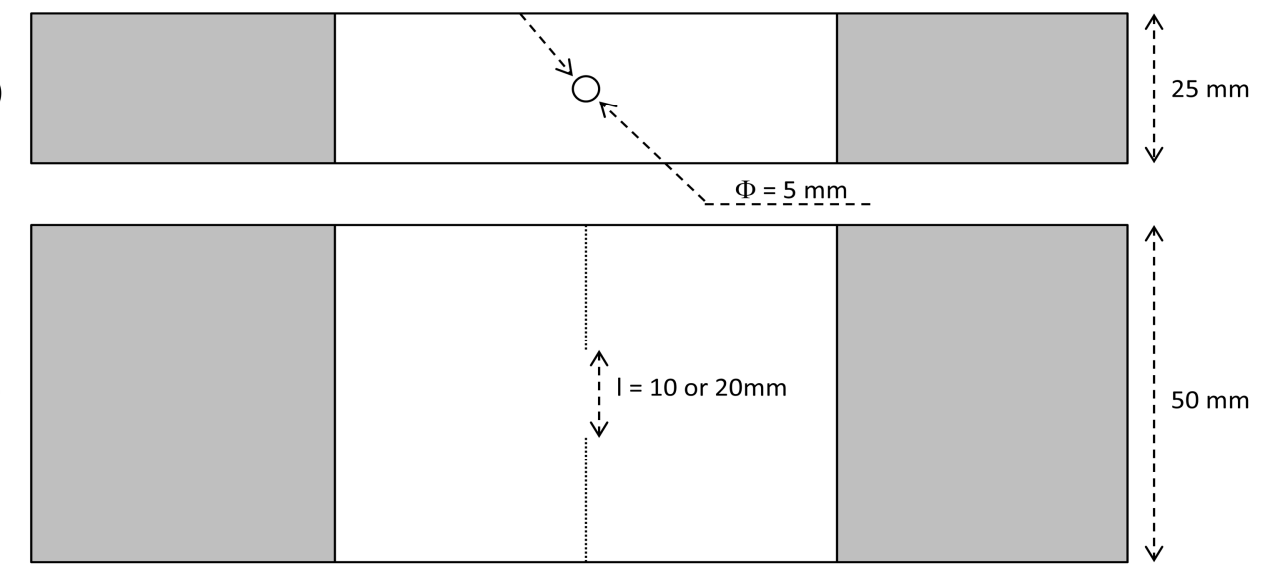

Fig. 1. Geometry of tensile specimens: (a) unnotched, (b) open-hole and (c) DENT

\subsection{Digital Image Correlation processing}

Many methods provide displacement or strain maps on deformed surfaces (digital image correlation, photoelasticity, moiré interferometry, speckle interferometry, etc.). Among them, the Digital Image Correlation (DIC) method is probably one of the most popular due to its implementation ease and the development of commercial softwares.

Figure 2 shows the scheme of a mechanical test with the DIC device used in this study, where the focal axis of the camera needs to be centered and orthogonal to the sample. Without going into details, the implementation of the DIC method involves the following steps: 
- Creation of an artificial speckle: a black fine pencil was used to create a random and contrasted speckle;

- Lighting of the specimen: a pair of cool white lights have been used to prevent the material from heating;

- Focus adjustment;

- Acquisition: images were acquired from the beginning to the end of the test with an acquisition frequency of $10 \mathrm{fps}$;

- Post-processing: the kinematic analysis is based on an incremental correlation (with an incremental calculation mode): each image is compared to the previous image rather than the reference image (this is more satisfactory in cases of pattern breakdown or extremely high strains, as in our case).

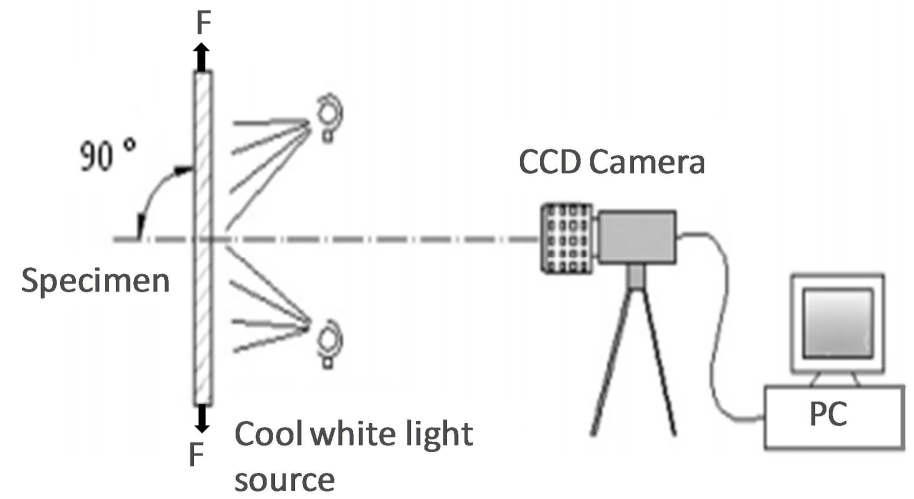

Fig. 2. Schematic experimental set-up

\section{Constitutive model}

\subsection{Context and objectives}

The 3-phase model which will be subsequently used for the numerical simulation has been introduced in an earlier study dealing with similar semi-crystalline polymer films [22]. It allowed to describe properly the different double yielding points encountered in both cases of uniaxial and biaxial drawing, for polyamide 6 and polyethylene films at $90^{\circ} \mathrm{C}$. It will be employed here for other polyethylene films at room temperature, in the more general context of non-uniform multi-axial stress/strain states, in order to show the ability of such a model to deal with polymer films of various geometries under arbitrary loading conditions. In the sequel, the main theoretical features of the 3phase model (based on physical considerations and experimental observations) are first recalled, together with the calibration procedure (see [22] for a more detailed presentation). Then, the following computations require the use of a finite element program and thus the preliminary numerical implementation of the model formulation in a calculation code, which appears to be the main challenge of this study and which will be therefore presented in details at the end of this section. 


\subsection{Theoretical formulation}

\subsubsection{Basic components of the 3-phase model}

Semi-crystalline polymers are merely composed of many crystalline blocks which are almost randomly distributed in an amorphous medium, so that the material appears to be almost homogeneous at the macroscopic scale. Owing to the lamellar structure of such materials, crystalline blocks are inter-connected by either very short intra-lamellar chains or inter-lamellar ones (standing for the amorphous phase) which are assumed to be either middle-sized or rather long. In the literature [31-33], semi-crystalline polymers are thus often viewed as 3-phase materials, including interfacial regions between successive purely crystalline and amorphous zones. In the present model, the three following constitutive behaviors are represented, according to the phase or the average distance between crystalline blocks.

\section{- Case 1}

When the distance between crystalline blocks is very short, most of the chains located between these blocks are connected or even embedded thereto. This first phase can thus be considered as purely crystalline and an elastoplastic constitutive model is retained in this case, with the von Mises criterion and a linear isotropic hardening, in the framework of generalized standard materials (the typical uniaxial stress-strain behavior is depicted in Figure 3(a) that displays the Young's modulus $E_{1}$, the tangent elastoplastic modulus $E_{T 1}$ and the associated yield stress $\sigma_{1}$ ).

\section{- Case 2}

When the distance between crystalline blocks is medium-sized, most of the chains are only partially connected to the surrounding blocks, in such a way that the mechanical response of the corresponding phase is rather governed by the presence of numerous entanglements between couples of chains.

In this case, the typical mechanical behavior under uniaxial tension is expressed as shown in Figure 3(b), displaying a new Young's modulus $E_{2}$, a new yield stress $\sigma_{2}$ and perfect plasticity. The maximum admissible stress $\sigma_{2}$ is proportional to the limit force corresponding to the incipient slippage between two arbitrary entangled chains under stretching, and also to the density of entanglements involved in the uniaxial behavior.

In multi-axial conditions, a modified von Mises equivalent stress is defined as follows:

$$
\sigma_{V M}^{\prime}=\frac{\sigma_{V M}}{K}
$$

where $\sigma_{V M}$ refers to the classical von Mises criterion, which has been retained as the sliding phenomenon between entangled chains is supposed to be governed by shear effects and distortion, and $K$ is a multi-axial factor specially defined so as to take into account the change in the number of 
entanglements brought into play with the deformation mode (in any multi-axial state, a new threshold stress may be defined which would be proportional to the sum of all these entanglements, regardless of their orientation, hence the expression (1) of the modified equivalent stress).

According to simple geometric considerations, $K$ is defined as the scaled sum of the positive principal strains $\varepsilon_{I}$ which are the ones and only strains in the principal axes that contribute to the interlocking of entanglements:

$$
K=\frac{\sum_{I} \varepsilon_{I}}{\max _{I} \varepsilon_{I}} \quad\left(\varepsilon_{I} \geq 0\right)
$$

This multi-axial factor $K$ evolves continuously and even linearly between $K=1$ and $K=2$ corresponding to the two limit cases of uniaxial and equi-biaxial states, respectively.

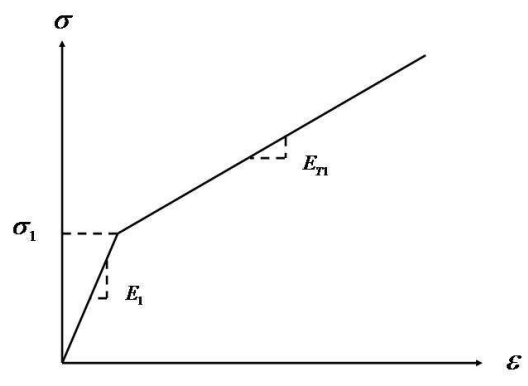

(a) Case 1

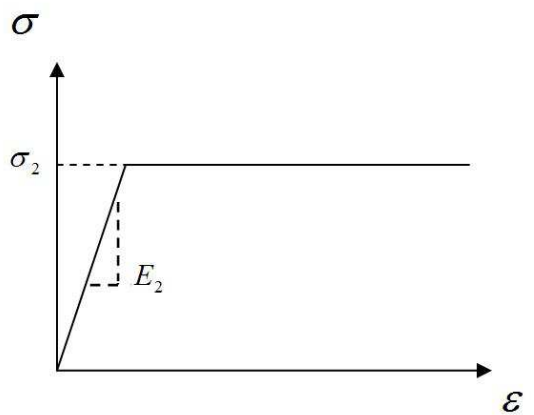

(b) Case 2

Fig. 3. Stress-strain curves under uniaxial tension

\section{- Case 3}

When the distance between crystalline blocks is long enough, the chains located between these blocks are mostly unconstrained. In these conditions, a network model such as the 8-chain model is a satisfactory solution to simulate the macroscopic mechanical behavior. This model is based on the so-called Langevin model introduced in the 1940's, in the context of non-Gaussian statistics, in order to deal with large stretches in amorphous materials up to the limiting end-to-end distance given by the length of the chains $[2,3]$. It is characterized by two material parameters, namely the density $C_{R}$ and the limit of extensibility of a chain $N$ [34].

\subsubsection{Full model}

The global constitutive response of the material is interpreted as follows in a 3D framework. At the macroscopic scale, the semi-crystalline polymers considered are supposed to be homogeneous and roughly isotropic. The global model encompassing the three behaviors presented above is 
depicted in Figure 4, in the style of a rheological model. Models for cases 1 and 2 are assumed to bear the same stress so that a series arrangement is retained. The model for case 3 initially corresponds to a much softer behavior which is paralleled with the others. This configuration has shown to be the most natural and adequate representation, capable to reproduce the predominance of either phase according to the deformation range. The mechanical behavior of the full model can then be deduced from the constitutive laws of the three different phases, by combining strain and stress tensors additively.

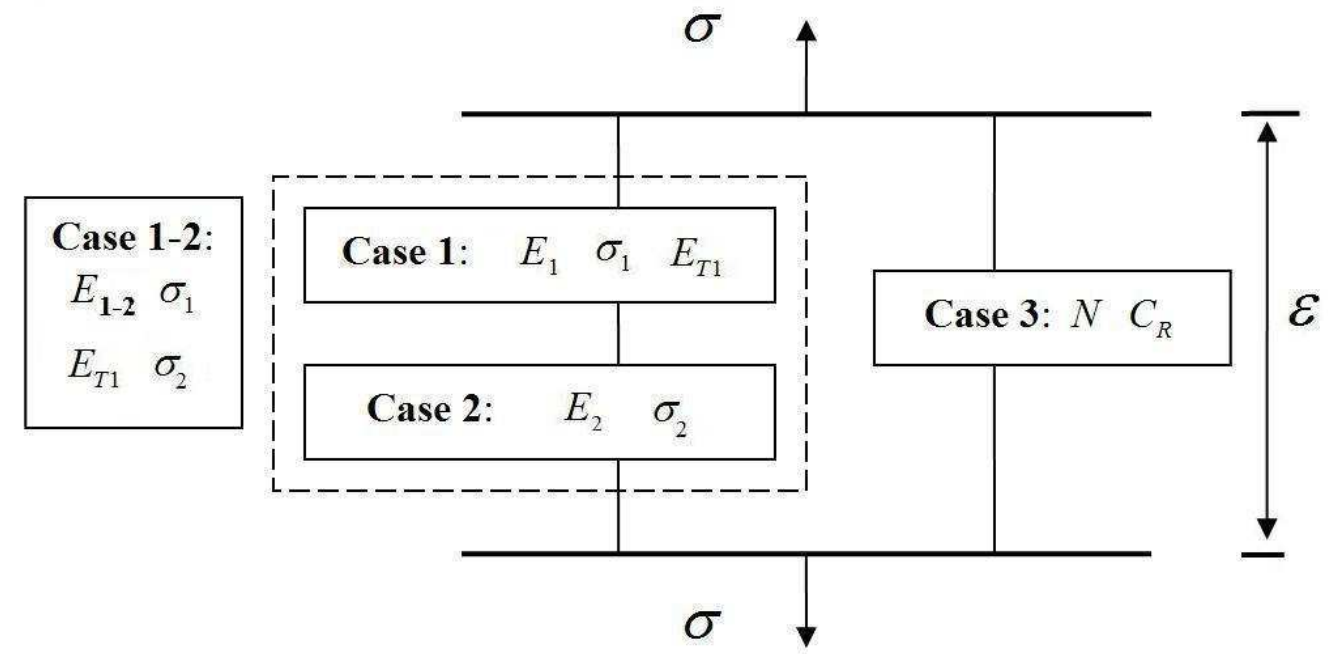

Fig. 4. Global representation of the mechanical model for semi-crystalline polymers

Initially developed on the basis of a constitutive behavior divided in three distinct parts, in practice, the final model turns out to rely ultimately on only two sub-models associated to the two branches in parallel in the rheological scheme. Indeed, dealing with the elastoplastic components (cases 1 and 2), both elastic behaviors are not particularized and a unique Young's modulus $E_{1-2}=E_{1} E_{2} /\left(E_{1}+E_{2}\right)$ is used for the serial arrangement (renamed case 1-2), in the framework of the Saint-Venant-Kirchhoff linear elastic theory. Dealing now with plasticity, a single skate component is used in order to represent in a unified way the two plastic cases in series configuration.

Let us mention finally that this model will be implemented within the context of a plane stress formulation, for thin films applications. It means that the global stress tensor must verify the plane stress condition. For simplicity purposes, we assume that both stress tensors (in cases 1-2 and 3) have a null transverse normal component, which is satisfied in practice.

\subsection{Calibration of the model parameters}

The model parameters for a given material are determined from a uniaxial tensile test and the corresponding experimental stress/strain curve (see Figure 5). Due to the very large strains encountered, true logarithmic (Hencky) strains are employed, as they are recognized to be the most 
physical strain measure [35]. These Hencky strains are additively decomposed into elastic and plastic parts, according to the classical Lee multiplicative decomposition of the deformation gradient. Kirchhoff stresses represent in principle the most reliable counterpart of logarithmic strains. In the present case, as volumetric changes are proved to be negligible, they practically coincide with the more classical true Cauchy stresses, which can thus also be employed for the description of the constitutive law.

The first two parts of the curve (for $0 \leq \varepsilon \leq \varepsilon_{A}$ and $\varepsilon_{A} \leq \varepsilon \leq \varepsilon_{B}$, respectively) are assumed to be linear, and their respective slopes $E$ and $H$ are obtained by linear regression. For $\varepsilon \geq \varepsilon_{B}$ in the vicinity of $\varepsilon_{B}$ (while strains remain moderate), the response curve is also quite linear, so that a third slope $E_{0}$ can be deduced again, which corresponds to the initial elastic modulus of case 3 . The set of elastoplastic coefficients (case 1-2) is then derived using the following relations:

$$
\begin{gathered}
E_{1-2}=E-E_{0} \\
E_{T 1}=H-E_{0} \\
\sigma_{1}=\sigma_{A}-E_{0} \varepsilon_{A} \\
\sigma_{2}=\sigma_{B}-E_{0} \varepsilon_{B}
\end{gathered}
$$

where $\sigma_{A}$ and $\sigma_{B}$ represent the limit stresses on the uniaxial curve, separating the three successive linear parts and associated with strains $\varepsilon_{A}$ and $\varepsilon_{B}$, respectively (these stresses have not been reported in Figure 5 for clarity purposes). The three slopes (and thus the subsequent moduli and yield stresses) have been obtained with a rather reasonable correlation coefficient. In view of this, and thanks to the very low level of scattering in the uniaxial stress-strain experimental curves, the elastoplastic properties are identified in a representative and repeatable manner, with very low uncertainty.

Afterwards, the maximum strain $\varepsilon_{\lim }$ and the corresponding limit stretch value $\lambda_{\text {lim }}=\exp \left(\varepsilon_{\text {lim }}\right)$ are estimated by extrapolation of the uniaxial curve, and $N$ is simply obtained as follows [36]:

$$
N=\frac{1}{3}\left[\lambda_{\lim }^{2}+\frac{2}{\lambda_{\lim }}\right]
$$

Using an arbitrary point $\left(\varepsilon_{t}, \sigma_{t}\right)$ on the stress-strain curve, with $\lambda_{e q}=\exp \left(\varepsilon_{t}\right)$ and $\sigma_{e q}=\sigma_{t}-\sigma_{2}$, one finally gets:

$$
C_{R}=\frac{\sigma_{e q}}{\lambda_{e q}^{2}-\frac{1}{\lambda_{e q}}} \sqrt{\frac{\lambda_{e q}^{2}+2 / \lambda_{e q}}{3 N}}\left[\mathscr{L}^{-1}\left(\sqrt{\frac{\lambda_{e q}^{2}+2 / \lambda_{e q}}{3 N}}\right)\right]^{-1}
$$


The limit of extensibility of a chain $N$ is proved to have little influence on the simulation results, as soon as it is sufficiently high (in a range of large values of $N, \varepsilon_{\lim }$ does not depend much on its specific value). By contrast, the density $C_{R}$, whose identification is somewhat hazardous, will have a strong influence on the simulation results, as it will be discussed when validating the model in uniaxial drawing.

According to experimental observations [22], the global behavior is assumed to be almost incompressible. Indeed, when large elastoplastic strains are involved, both models in parallel (1-2 and 3) display such a behavior. On one hand (case 1-2), the elastic strains are negligible, and the von Mises type criteria used imply an incompressible plastic behavior. On the other hand, the 8-chain model is consistent with this hypothesis. However, for particularly small strains, the global behavior is governed by the elastic part of case 1-2, which is represented by a Saint-Venant-Kirchhoff's law. A Poisson coefficient is thus needed for the model and it can be simply derived as the ratio between transverse and longitudinal strains in the onset of the uniaxial test. The effect of this Poisson's ratio can yet be neglected in most of the strain range considered, since it only concerns very small strains. What is more, the numerical value obtained is generally not so far from 0.5 , which means that these elastic strains are also nearly incompressible. Anyway, the apparent contradiction between a slightly compressible material and a perfectly incompressible model in parallel is not an issue since only bidimensional strains and stresses are further considered within the plane stress formulation retained.

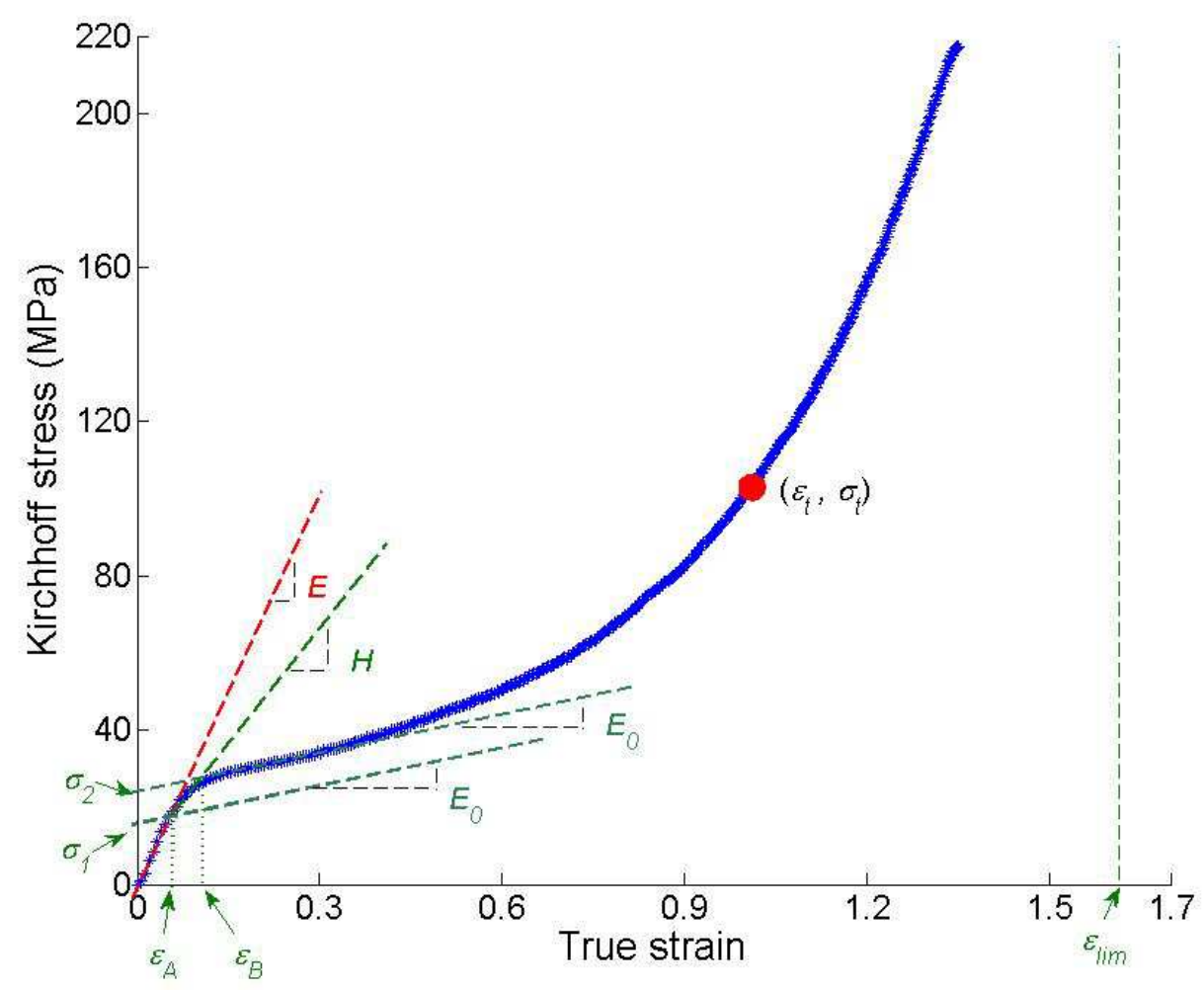

Fig. 5. Calculation of the model parameters using the uniaxial curve 


\subsection{Numerical implementation}

The 3-phase model discussed above is then implemented in the commercial finite element software ABAQUS through a User Material (UMAT) subroutine, so as to deal with complex geometries and/or loadings. In further applications, the polymer films are inherently bidimensional (exceptionally thin) and only submitted to in-plane loads, so that the strain and stress states are practically uniform across the thickness. In such conditions, only 2D models can be considered, verifying the plane stress assumption.

In the sequel, due to the high geometric and material non-linearities encountered, the problems are solved in an incremental way, using the Newton-Raphson iterative procedure, within the framework of an updated Lagrangian formulation. At each new iteration, a local integration is performed at each integration point so as to update the elastic/plastic strains and stresses, but also compute the consistent tangent constitutive tensor. The derivation of all these quantities from a given strain increment happens to be the main stumbling block of the numerical implementation.

Due to the parallel configuration of the 3-phase model, the two sub-models (cases 1-2 and 3) are first treated separately, and the respective stresses and tangent tensors are then added so as to deal with the full model.

\subsubsection{Elastoplastic behavior}

Using ABAQUS standard notations, the elastic tangent tensor writes classically as follows, in a 3D framework:

$$
\frac{\partial \boldsymbol{\sigma}}{\partial \boldsymbol{\varepsilon}}=2 G \mathfrak{J}+K \mathbf{I} \otimes \mathbf{I}
$$

where $G$ and $K$ are the shear and bulk moduli, and $\mathbf{I}$ and $\mathfrak{J}$ stand for the second-order and fourth-order unity tensors, respectively $\left(\mathfrak{I}_{i j \mathrm{jl}}=\delta_{i l} \delta_{k j}\right.$ where $\delta_{i j}$ is the Kronecker symbol). The stress and strain tensors will be further decomposed in their deviatoric and spherical parts as follows:

$$
\boldsymbol{\varepsilon}=\mathbf{e}+\frac{1}{3} \varepsilon_{v o l} \mathbf{I} \quad \boldsymbol{\sigma}=\mathbf{S}-p \mathbf{I}
$$

In the framework of generalized standard materials (with only isotropic hardening), the von Mises criterion writes:

$$
q=\bar{\sigma}\left(\bar{e}^{p l}\right)
$$

involving the von Mises stress $q$ and the yield stress $\bar{\sigma}$ depending on the equivalent plastic strain $\bar{e}^{p l}$ (which is inherently deviatoric). The plastic strain increment is obtained from the evolution law which is written in a discretized way, according to the implicit Euler time integration scheme:

$$
\Delta \mathbf{e}^{p l}=\left.\mathbf{e}^{p l}\right|_{t+\Delta t}-\left.\mathbf{e}^{p l}\right|_{t}=\frac{3}{2} \Delta \bar{e}^{p l} \frac{\mathbf{S}}{q}
$$


Inserting Equation (12) in the elastic constitutive relation at current time and eliminating the stress tensor (by using criterion (11)) leads to a non-linear equation in $\Delta \bar{e}^{p l}$ which is solved in an iterative manner. Once $\Delta \bar{e}^{p l}$ is known, the computation of stresses and plastic strains is straightforward. Finally, the consistent elastoplastic tangent tensor (depending on the increment size) can be obtained by differentiating the previous stress-strain relation at current time (see [37] for more details):

$$
\frac{\partial \boldsymbol{\sigma}}{\partial \boldsymbol{\varepsilon}}=Q \mathfrak{J}+\left(K-\frac{1}{3} Q\right) \mathbf{I} \otimes \mathbf{I}-R \mathbf{S} \otimes \mathbf{S}
$$

with:

$$
Q=\frac{2}{3} \frac{q}{\tilde{e}} \quad R=\frac{1}{q \tilde{e}} \frac{1-\Delta \bar{e}^{p l} H / q}{1+H / 3 G}
$$

where $\tilde{e}=\sqrt{\frac{2}{3} \hat{\mathbf{e}}: \hat{\mathbf{e}}}\left(\hat{\mathbf{e}}=\left.\mathbf{e}^{e l}\right|_{t}+\Delta \mathbf{e}\right)$ and $H=\frac{d \bar{\sigma}}{d \bar{e}^{p l}}$ is the hardening modulus.

The radial return mapping algorithm, presented above in the 3D framework, becomes more complex when considering the plane stress assumption, as the solution obtained with the $3 \mathrm{D}$ procedure does not verify a priori the plane stress condition and must be conveniently projected into the plane stress space. In practice, the normal out-of-plane strain increment can be expressed in terms of the in-plane strains by using the plane stress condition. It allows one to reduce the previous elastoplastic tangent tensor (13) to a new expression involving the 2D reduced tensors (among which the in-plane strains $\underline{\boldsymbol{\varepsilon}}$ and stresses $\underline{\boldsymbol{\sigma}}$ ) classically written in matrix notation:

$$
\frac{\partial \underline{\boldsymbol{\sigma}}}{\partial \underline{\boldsymbol{\varepsilon}}}=\left[Q \underline{\mathfrak{J}}+\left(K-\frac{1}{3} Q\right) \underline{\mathbf{I}} \otimes \underline{\mathbf{I}}-R \underline{\mathbf{S}} \otimes \underline{\mathbf{S}}\right]-P\left(\left(K-\frac{1}{3} Q\right) \underline{\mathbf{I}}+R p \underline{\mathbf{S}}\right) \otimes\left(\left(K-\frac{1}{3} Q\right) \underline{\mathbf{I}}+R p \underline{\mathbf{S}}\right)
$$

where:

$$
P=\frac{1}{K+\frac{2}{3} Q-R p^{2}}
$$

The presence of two elastoplastic models in series configuration does not modify the general numerical approach but only changes the hardening modulus value depending on the current stress level. Similarly, the multi-axial factor involved in the modified von Mises criterion (case 2) can easily be introduced in the above expressions without changing the calculation process.

\subsubsection{Hyperelastic behavior}

The stress and tangent tensors will be expressed here as functions of the strain energy which generally depends on the three classical invariants of the strain tensor (or its deviatoric part). When considering the 8-chain model, the strain energy can be naturally expressed in terms of only the first 
invariant $\bar{I}_{1}=\mathbf{I}: \overline{\mathbf{B}}$ of the left deviatoric Cauchy-Green deformation tensor $\overline{\mathbf{B}}=\overline{\mathbf{F}} \cdot \overline{\mathbf{F}}^{T}$, where $\overline{\mathbf{F}}=J^{-\frac{1}{3}} \mathbf{F}$ is the deviatoric part of the deformation gradient tensor $\mathbf{F}$. However, due to the incompressibility of the hyperelastic phase, the jacobian determinant $J=\operatorname{det}(\mathbf{F})$ equals unity so that the overlines may be removed in the sequel.

In the general 3D case, the variation of the strain energy density simply writes as follows:

$$
\delta U=J\left(\mathbf{S}: \delta \mathbf{e}-p \delta \varepsilon_{v o l}\right)
$$

using the same definitions as before for the spherical and deviatoric strains and stresses. According to the previous assumptions, and observing that $\delta I_{1}=\mathbf{I}: \delta \mathbf{B}=2 \mathbf{B}: \delta \mathbf{e}$, one can derive the deviatoric stress tensor by considering an arbitrary variation of the deviatoric strain $\delta \mathbf{e}$ :

$$
\mathbf{S}=2\left(\mathfrak{J}-\frac{1}{3} \mathbf{I} \otimes \mathbf{I}\right):\left[\frac{\partial U}{\partial I_{1}} \mathbf{B}\right]
$$

With the help of variational calculations (see [37] for more details), one can express the associated deviatoric tangent tensor as follows:

$$
\begin{aligned}
\frac{\partial \mathbf{S}}{\partial \boldsymbol{\varepsilon}} & =\frac{\partial \mathbf{S}}{\partial \mathbf{e}}:\left(\mathfrak{I}-\frac{1}{3} \mathbf{I} \otimes \mathbf{I}\right)=2\left(\mathfrak{I}-\frac{1}{3} \mathbf{I} \otimes \mathbf{I}\right): \frac{\partial}{\partial \mathbf{e}}\left(\frac{\partial U}{\partial I_{1}} \mathbf{B}\right):\left(\mathfrak{I}-\frac{1}{3} \mathbf{I} \otimes \mathbf{I}\right) \\
& =2\left(\mathfrak{I}-\frac{1}{3} \mathbf{I} \otimes \mathbf{I}\right):\left(\frac{\partial U}{\partial I_{1}} \mathbf{H}+2 \frac{\partial^{2} U}{\partial I_{1}^{2}} \mathbf{B} \otimes \mathbf{B}\right):\left(\mathfrak{I}-\frac{1}{3} \mathbf{I} \otimes \mathbf{I}\right)
\end{aligned}
$$

with $\mathbf{H}: \delta \mathbf{e}=\delta \mathbf{e} \cdot \mathbf{B}+\mathbf{B} \cdot \delta \mathbf{e}$, namely $H_{i j k l}=\frac{1}{2}\left(\delta_{i k} B_{j l}+B_{i k} \delta_{j l}+\delta_{i l} B_{j k}+B_{i l} \delta_{j k}\right) . \quad$ Conversely, $\delta \varepsilon_{v o l}=0$ so that the equivalent pressure (which appears in Equation (17)) can not be directly determined in the same way.

Under the plane stress assumption, the strain and stress measures may be reduced to 2D tensors (which will be underlined in the sequel), excluding thus the normal out-of-plane component (in $z$-direction). In this case, the equivalent pressure simply writes $p=S_{z z}$ and the in-plane stress components can be derived from the preceding 3D expression of the deviatoric tensor (18):

$$
\begin{gathered}
\sigma_{x x}=S_{x x}-p=2 \frac{\partial U}{\partial I_{1}}\left(B_{x x}-B_{z z}\right) \\
\sigma_{y y}=S_{y y}-p=2 \frac{\partial U}{\partial I_{1}}\left(B_{y y}-B_{z z}\right) \\
\sigma_{x y}=2 \frac{\partial U}{\partial I_{1}} B_{x y}
\end{gathered}
$$


Finally, $B_{z z}$ can be deduced from the incompressibility condition $\operatorname{det}(\mathbf{B})=1$ :

$$
B_{z z}=\frac{1}{\operatorname{det}(\underline{\mathbf{B}})}
$$

so that the tangent tensor takes the following form:

$$
\frac{\partial \underline{\boldsymbol{\sigma}}}{\partial \underline{\boldsymbol{\varepsilon}}}=2\left[\frac{\partial U}{\partial I_{1}} \underline{\mathbf{H}}+2 \frac{\partial U}{\partial I_{1}} B_{z z} \underline{\mathbf{I}} \otimes \underline{\mathbf{I}}+2 \frac{\partial^{2} U}{\partial I_{1}^{2}}\left(\underline{\mathbf{B}}-B_{z z} \underline{\mathbf{I}}\right) \otimes\left(\underline{\mathbf{B}}-B_{z z} \underline{\mathbf{I}}\right)\right]
$$

with $\underline{H}_{i j k l}=\frac{1}{2}\left(\delta_{i k} \underline{B}_{j l}+\underline{B}_{i k} \delta_{j l}+\delta_{i l} \underline{B}_{j k}+\underline{B}_{i l} \delta_{j k}\right)$.

\section{Results: calibration, validation and discussion}

\subsection{Calibration of the model parameters in uniaxial drawing}

First and foremost, the experimental tensile tests performed on unnotched specimens are used for calibration purposes. The procedure discussed above is applied to the corresponding stressstrain curve (one single curve is arbitrarily retained for the calibration, since all the tested specimens give very similar results). The following parameters are identified: $\sigma_{1}=5.53 \mathrm{MPa}, \sigma_{2}=10 \mathrm{MPa}$, $E_{1-2}=207.9 \mathrm{MPa}, N=18.3, C_{R}=2.45 \mathrm{MPa}, E_{T 1}=122.4 \mathrm{MPa}$ and $v=0.4$.

Once the model parameters are known, they can be introduced in the finite element program. The uniaxial drawing of a rectangular specimen is then simulated (the dimensions of the specimen are chosen arbitrarily, since the uniform stress-strain response does not depend on the geometry). Furthermore, only one rectangular linear finite element is used (with as few nodes as possible and reduced integration), for efficiency purposes.

A very good agreement between the experimental and numerical results is naturally expected, since material parameters were chosen in order to closely fit the uniaxial test response. Figure 6 displays both numerical and experimental curves, for validation purposes. From small up to moderate strains, the simulation results really fit the experimental ones very well. When the strains become much larger, the two curves slightly differ due to the strain softening phenomena. In the present case, the arbitrary point $\left(\varepsilon_{t}, \sigma_{t}\right)$ used for the calibration of the 8-chain model has been chosen in the first half of the curve. The value of parameter $C_{R}$ has proved to be the same whatever the point in that particular range, and it gives rise to a numerical curve nearly coincident with the experimental one as long as moderate strains are considered. In the following applications, the strain level is such that no special effort has been made in order to better match the experimental and numerical results in very large strains. 


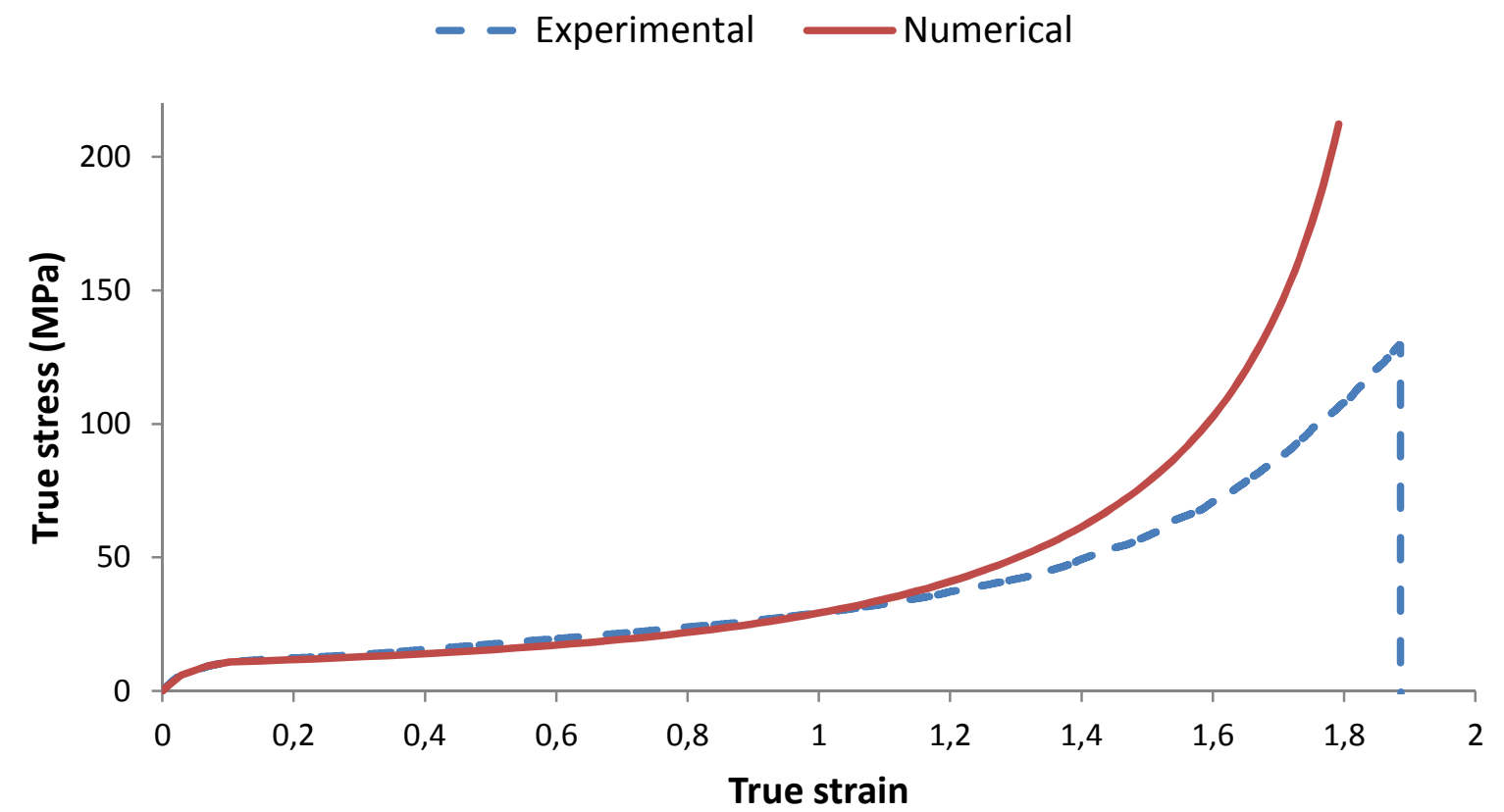

Fig. 6. Comparison between experimental and simulation results in uniaxial drawing

\subsection{Open-hole specimens under uniaxial tension}

\subsubsection{Experimental results}

Four different specimens (with various thicknesses) have been stretched in the longitudinal direction. During the mechanical tests, the global tensile force and the length variation (maximum displacement in the longitudinal direction) are recorded: the corresponding force-displacement curves are plotted in Figure 7 (for comparison purposes, the force was divided by the current thickness).

The same typical behavior is observed for all the specimens. In the first stage of deformation, the force-displacement curve is almost linear, until it reaches a limit value (corresponding to the incipient plasticity). At such points, a few oscillations occur, with successive force peaks and subsequent slight decreases. These oscillations identify with a kind of Piobert-Lüders plateau and the corresponding mechanical response can be viewed as a necking phenomenon whose initiation would be facilitated by the presence of the hole in the specimen, owing to the non uniform deformations throughout the specimen. Then, the force increases again and finally reaches something like a plateau, where the force hardly changes until collapse. Large discrepancies are noticed between the different limit force values, even when reduced to a common thickness (with a maximum relative difference of about $20 \%$ ). These discrepancies are naturally due to unavoidable imperfections related to the geometry (thickness variations, size and position of the central hole) or the boundary conditions. 


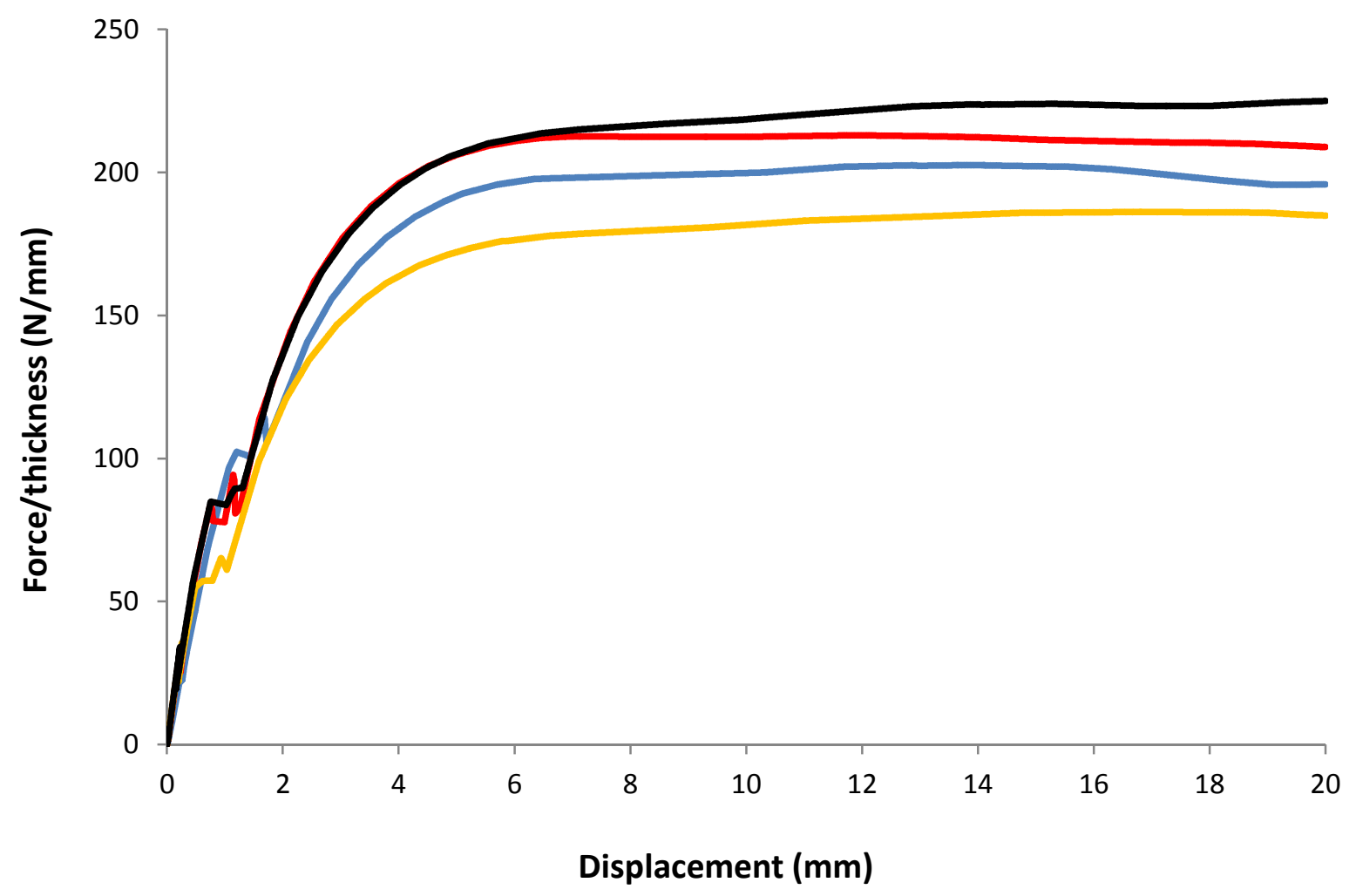

Fig. 7. Experimental force-displacement curves of open-hole specimens under uniaxial tension

The displacement fields within the specimens have been recorded during the tensile tests using the DIC method, though the corresponding strain distributions will only be displayed later, together with the simulation results for comparison purposes.

\subsubsection{Modeling hypotheses}

The model used for the numerical computations and displayed in Figure 8 only involves one quarter of the central part of the open-hole specimen, for obvious reasons of symmetry. The thickness of one of the tested specimens is arbitrarily retained for the calculations, namely $70 \mu \mathrm{m}$. Symmetry conditions are naturally applied onto the two cut edges. The longitudinal external edge is free as well as the circular edge around the hole. The end of the specimen is submitted to a uniform enforced displacement, in accordance to the experimental loading conditions. The simulations are carried out up to a maximum displacement of $10 \mathrm{~mm}$. It corresponds to a global elongation of $20 \mathrm{~mm}$ for the entire specimen, which is roughly the maximum value reached experimentally before failure. 


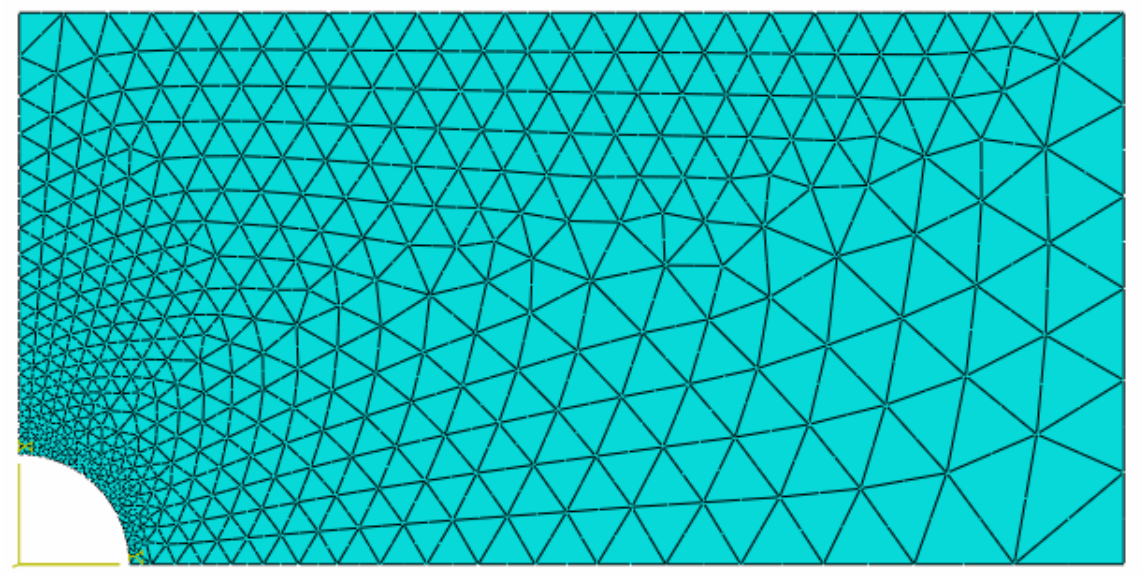

Fig. 8. Geometric model and mesh for the open-hole tensile tests

Two-dimensional 6-node triangular finite elements are used, with quadratic shape functions and full integration (namely 3 integration points per element). Preliminary convergence tests have been performed and the mesh depicted in Figure 8 was finally obtained. It is particularly fine in the vicinity of the circular hole, where stresses show the greatest variations, and coarser anywhere else, as stresses become more and more uniform as one moves away from the central hole. Particular attention is paid to the regularity of the mesh, especially around the hole, where excessive distortion may appear in finite strains.

For comparison purposes, two simulations are performed. Whereas the first one naturally involves the 3-phase model previously presented, the second one considers a standard elastoplastic model instead, which is based on the classical von Mises criterion and an isotropic hardening. The corresponding moduli and yield stresses are identified thanks to the same experimental uniaxial curve (with similar linear regressions).

\subsubsection{Validation and discussion}

The simulation results are first confronted to the experimental ones through the forcedisplacement curves. In Figure 9, the global force required to stretch the global specimen is plotted versus the corresponding longitudinal elongation of the full specimen. The four experimental curves of Figure 7 are redrawn and the two simulation curves (corresponding to the 3-phase and standard models, respectively) are added for validation purposes.

All the curves perfectly coincide during the elastic stage. Thereafter, the agreement between the simulation curve associated to the 3-phase model and the set of experimental curves is quite acceptable. The general shape of the curve is very similar (except for the oscillations, corresponding to incipient plasticity, which are not represented by the present model) and the limit admissible force (before failure) predicted by the numerical computation lies in the interval of the stabilized forces deduced from all the experimental tests. 


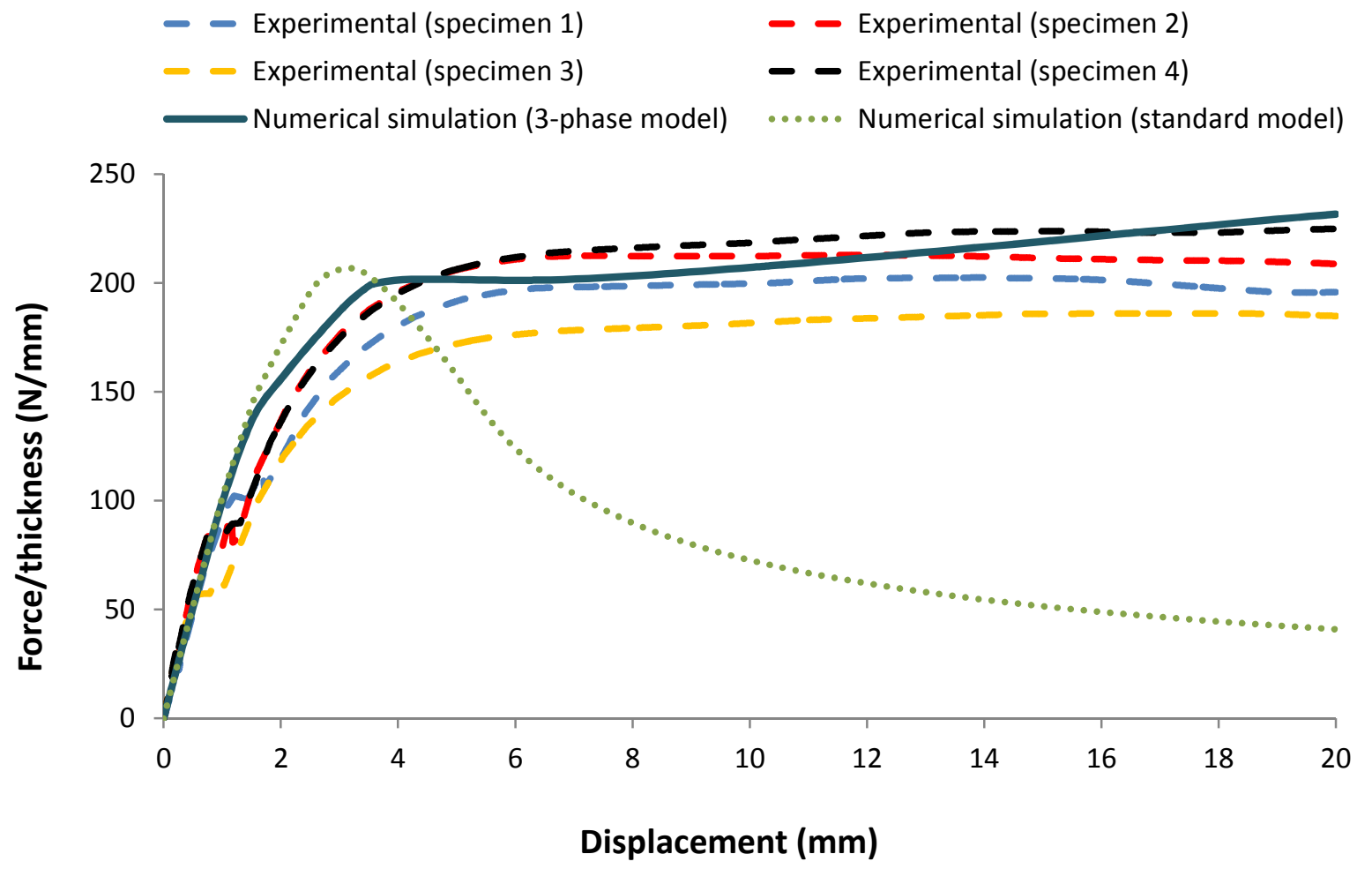

Fig. 9. Comparison between experimental and numerical results of open-hole specimens under uniaxial tension

By contrast, the simulation curve related to the standard model is totally different. A peak force is first noticed at a force level similar to the limit forces previously discussed. Then, a strong drop is observed so that the calculated force falls down to no more than $25 \%$ of the experimental values. The large discrepancies between the simulation curves that essentially occur in large strains are certainly due to the two main differences between the two constitutive models in hand. The presence of the 8-chain component in the 3-phase model is surely responsible for the higher strength of the material in very large strains. But it is worth mentioning that at such strain levels, both 3-phase and standard models are equally able to reproduce the uniaxial response of unnotched specimens (when properly calibrated). Actually, the best explanation for these discrepancies is due to the multiaxial nature of strains and stresses in the open-hole specimen. Even for moderate strains, the 3phase model is better able to describe the experimental behavior due to the favorable influence of the multi-axial factor. This analysis confirms the high quality of our 3-phase model and the relative importance of each phase in such a multi-axial context.

Figure 10 depicts Hencky strain distributions in the longitudinal direction at two particular loading levels, obtained both experimentally (with the DIC method) and numerically (with the finite element model). Similar color scales have been applied in both experimental and numerical graphs so as to best compare the contoured maps of the longitudinal strain. From a qualitative point of view, the numerical and experimental deformed shapes are very close to each other, and the numerical graphs compare well with the experimental ones (in terms of color pattern), especially in the most deformed state. The main difference lies in the vicinity of the hole. The very large strains obtained with the numerical calculations (depicted in red) are not observed experimentally since the 
post-processing calculations performed in the framework of the DIC method do not allow one to evaluate the strain values near the edges.

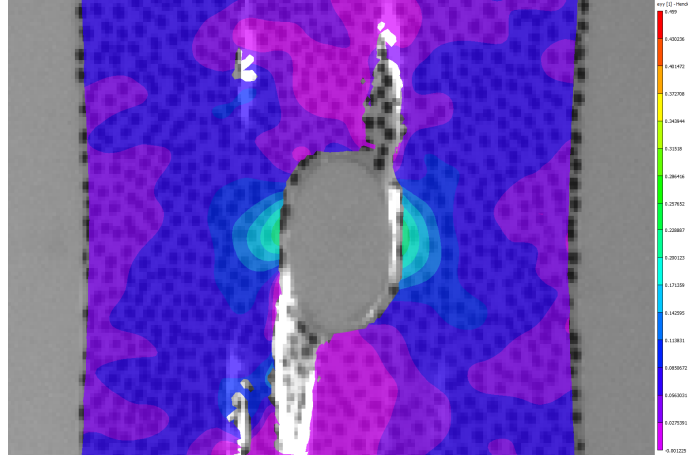

(DIC/maximum displacement $=3.216 \mathrm{~mm})$

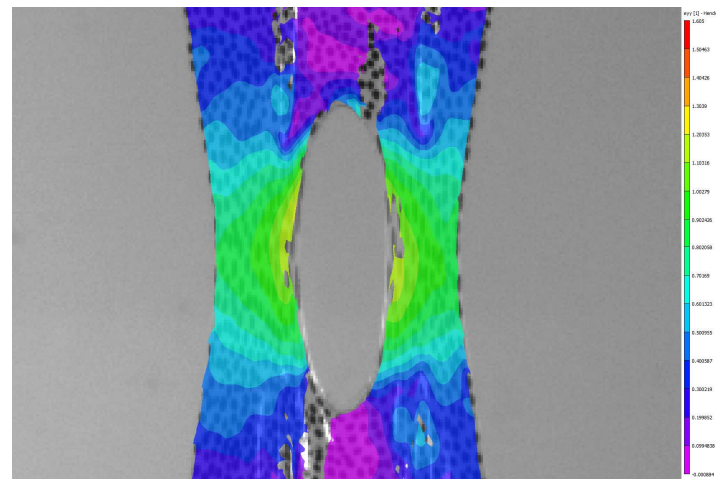

$(D I C /$ maximum displacement $=20 \mathrm{~mm})$

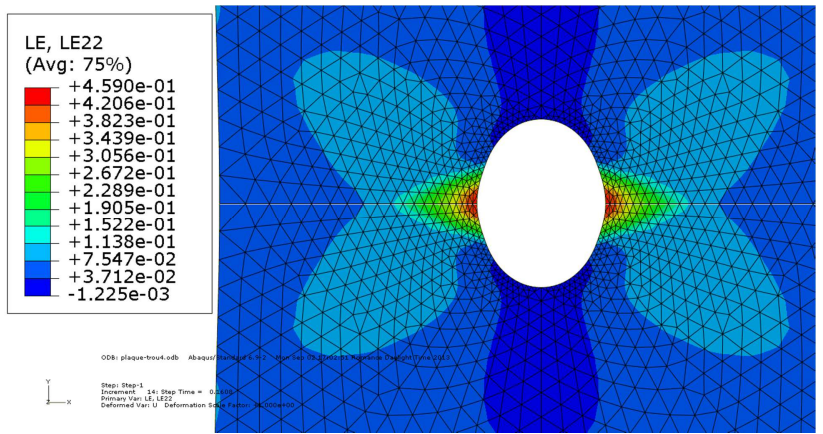

(FE/maximum displacement $=3.216 \mathrm{~mm})$

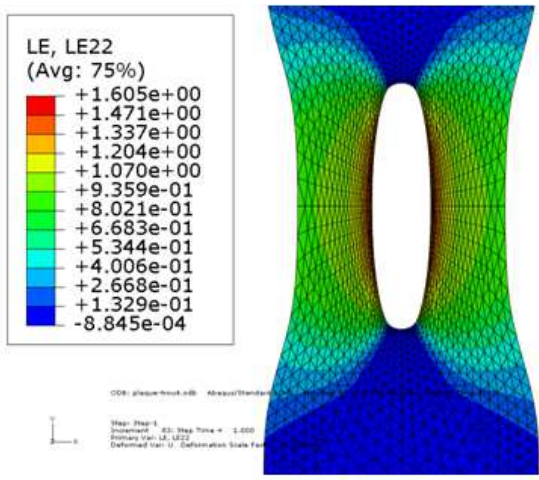

(FE/maximum displacement $=20 \mathrm{~mm}$ )

Fig. 10. Comparisons between DIC and FE results of open-hole specimens under uniaxial tension: longitudinal strain distributions for various enforced displacements

For a more quantitative comparison, the longitudinal Hencky strains have been plotted along the transverse symmetry axis, as seen in Figure 11 . The strain profiles obtained both experimentally and numerically are in very good accordance. At some distance from the hole, the results show a very good agreement with each other. When getting close to the hole, the experimental values either underestimate the numerical solution or are just not available.

Furthermore, as regards von Mises stresses, the maximum value appears constantly around the hole, specifically on the transverse symmetry axis, and the corresponding stress state is purely uniaxial in the longitudinal direction. What is noticeable is that this local stress is three times as large as the nominal longitudinal stress (which is uniformly obtained far from the hole), as observed analytically with an infinite plate in small strains and linear elasticity. 


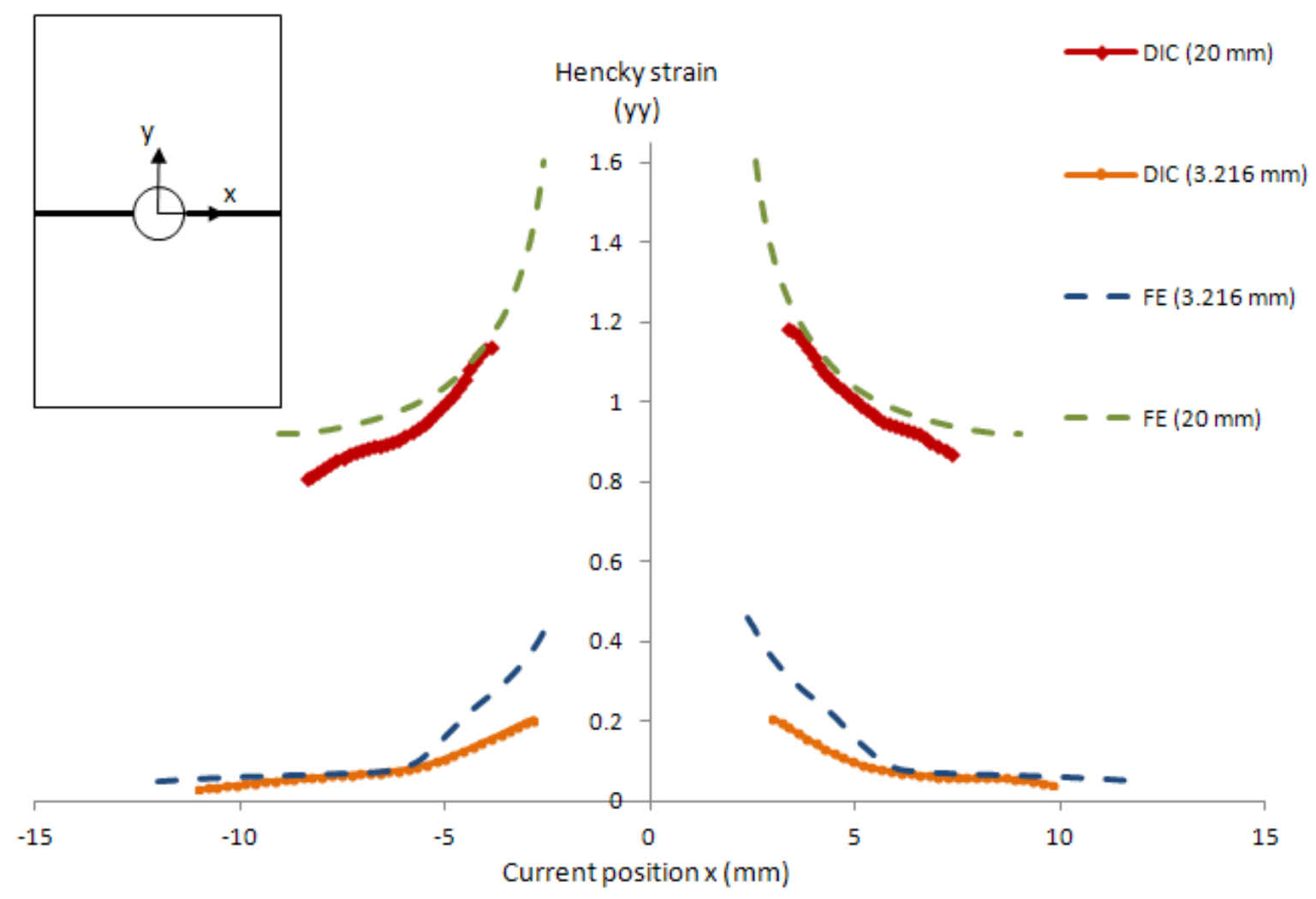

Fig. 11. Comparisons between DIC and FE results of open-hole specimens under uniaxial tension: longitudinal strain distributions for various enforced displacements (along the horizontal symmetry axis)

\subsection{Double Edge Notched Tensile (DENT) specimens}

\subsubsection{Experimental results}

Two different initial crack lengths have been considered during the experimental tests. In the first case where $I=10 \mathrm{~mm}$ (see Figure 1), the experimental force-displacement curve of an arbitrary specimen (whose thickness is $71 \mu \mathrm{m}$ ) serves as a reference for the calibration of the cohesive parameters which are here necessary for the description of the crack propagation. The plotted force and displacement still represent the global tensile force applied to the specimen and the corresponding length variation. Considering then the smallest initial crack length $(I=20 \mathrm{~mm}$ in Figure 1), two force-displacement curves are operated for validation purposes, corresponding to two specimens with different thicknesses (for comparison purposes, the force is divided by the current thickness). All these curves will be displayed in the next figures together with the simulation curves. Strain distributions measured experimentally using the DIC method will also be presented in the validation subsection. 


\subsubsection{Modeling hypotheses}

Among the possible alternative methodologies, the cohesive element method is used to model the crack propagation in the DENT specimens, as it is the most appropriate in this context where the crack path is known a priori. Let us focus on the remaining ligament which will be modeled by a cohesive zone whose width is arbitrarily set to $0.1 \mathrm{~mm}$ (which is identical to the width of the rectangular space representing the initial crack, see Figure 12). A specific constitutive behavior is required for this cohesive zone, whose criteria and evolution laws will be chosen among the ones available in ABAQUS software.

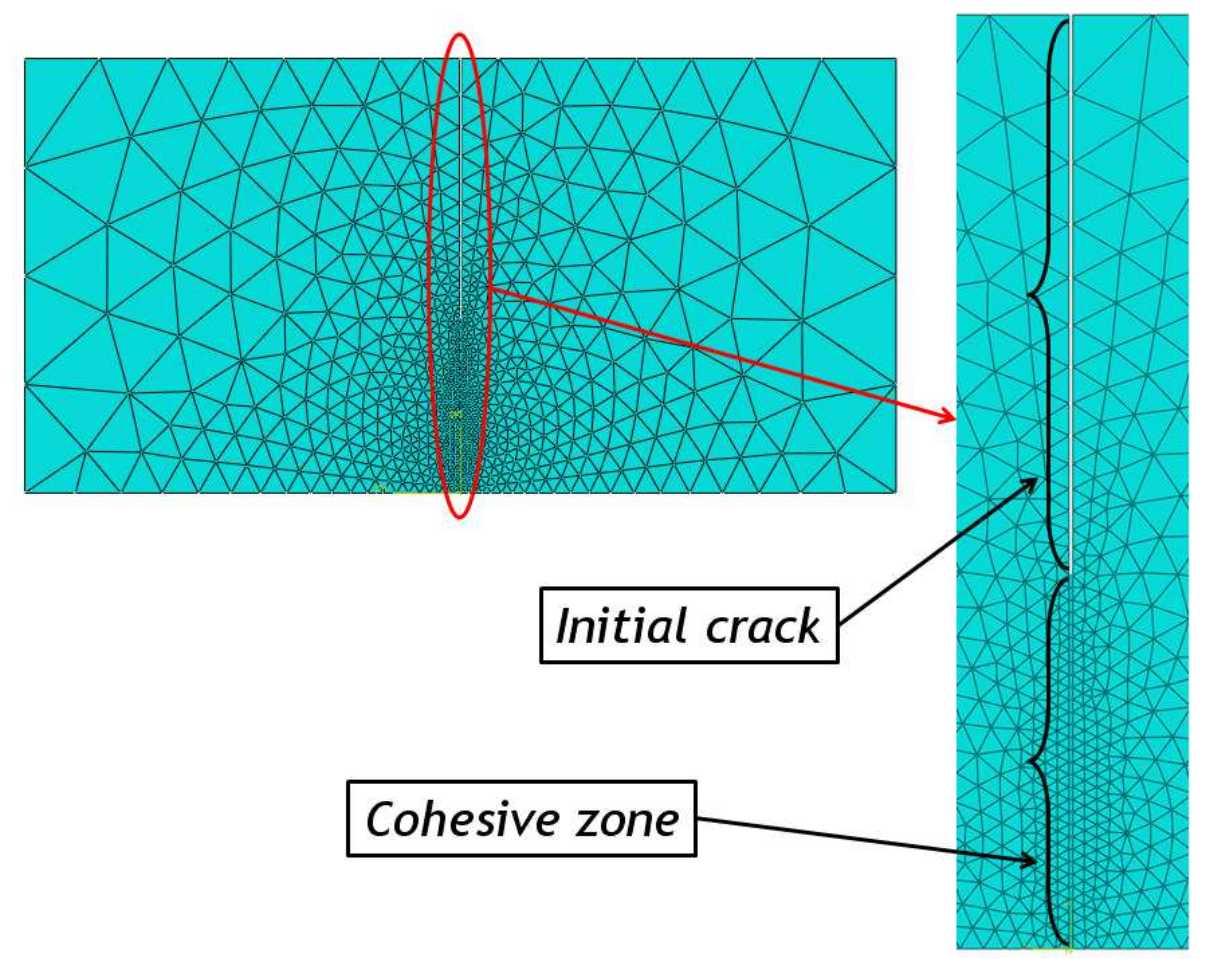

Fig. 12. Geometric model and mesh for the DENT tests

In practice, an elastic damage model is employed, regardless of the 3-phase model used for the rest of the specimen. The constitutive behavior of the cohesive elements can be schematically described by a set of traction-separation curves, as depicted in Figure 13, one for each direction. In each case, the nominal stress $t^{0}$ is expressed as a function of the associated (relative) displacement $\delta$. Here, due to the present geometric and loading configuration, the opening mode is highly dominating so much so that the tangential behavior will be ignored in the sequel and no multi-axial damage criterion will be needed, neither for the initiation nor for the evolution. The first part of the traction-separation curve is linear, characterized by an elastic modulus $E^{C}$, until the nominal stress reaches a maximum yield value $t_{n}^{0}$ corresponding to the critical displacement $\delta_{n}^{0}$. Damage initiates at this point and failure occurs when the stress vanishes, corresponding to the final displacement $\delta_{n}^{t}$ and the total dissipated energy $G^{C}$ (represented by the area under the traction-separation curve). A linear softening behavior is finally retained for the damage evolution law, so that the second part of the curve is also linear, as illustrated in Figure 13. 


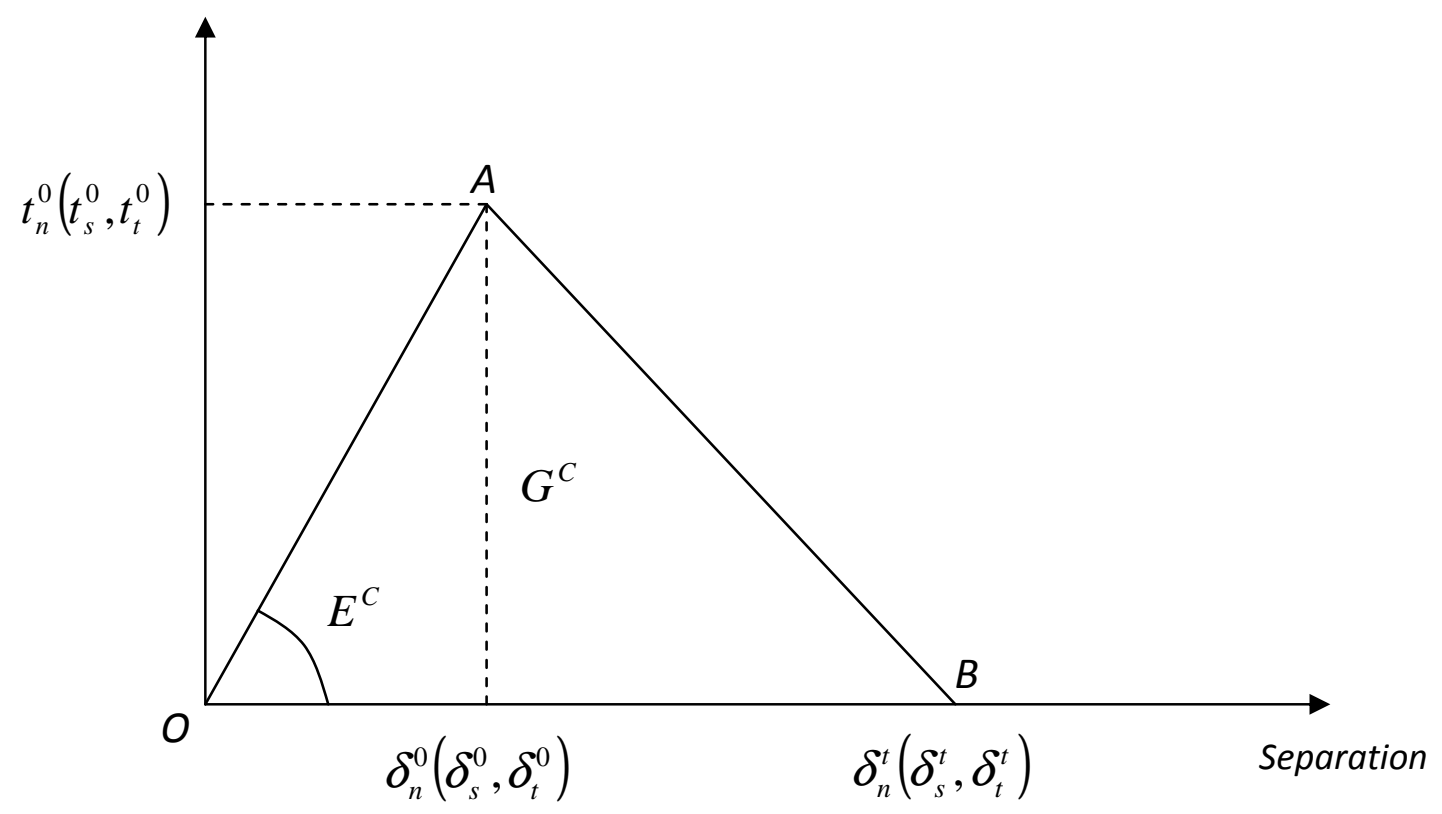

Fig. 13. Typical traction-separation curve of a cohesive element

The model used for the numerical computations only involves one half of the central part of the DENT specimens, for obvious reasons of symmetry. The thickness of one of the tested specimens is arbitrarily retained for each of the two calculations, namely $71 \mu \mathrm{m}$ for the calibration and $85 \mu \mathrm{m}$ for the validation case. Symmetry conditions are naturally applied onto the cut edge. The longitudinal external edges are free, on both sides of the crack. One end of the specimen is fixed while the other one is submitted to a uniform enforced displacement, in accordance to the experimental loading conditions. The simulations are carried out up to maximum displacements far beyond the experimental values corresponding to the final collapse of the specimens.

The rectangular cohesive zone (where the crack propagates) is meshed with one layer of 4node rectangular cohesive elements (with bi-linear interpolation), so that the mesh of the remaining part of the specimen is made of 3-node linear triangular finite elements, for compatibility purposes. The final mesh is particularly refined in the vicinity of the initial crack tip and along the crack path, where stresses supposedly show the most important variations and excessive distortion is expected in finite strains.

\subsubsection{Calibration of the cohesive parameters}

The experimental curve plotted in Figure 14 (with $I=10 \mathrm{~mm}$ ) serves as a reference for the calibration of the cohesive properties. Only three new parameters are needed in order to describe the cohesive behavior discussed above. First, the elastic modulus $E^{C}$ is set to $207.9 \mathrm{MPa}$, which corresponds to the initial elastic modulus previously obtained, in order to comply with the 3-phase model definition. Anyway, this parameter was found to have no particular effect on the global results. The maximum stress $t_{n}^{0}$ greatly affects the crack propagation "speed", whereas it has 
virtually no influence on the maximum global force in the force-displacement curve. By minimizing the difference between the experimental and numerical curves in a least square sense, one obtains the optimal value $t_{n}^{0}=25 \mathrm{MPa}$. The failure energy $G^{C}$ must be necessarily higher than the elastic strain energy involved in the first linear part of the traction-separation law. Conversely, excessive energy values may lead to convergence problems, due to an important distortion of the elements adjacent to the cohesive zone. Within a range of intermediate values, the energy does not influence so much the force-displacement response, so that an average value of $4 \mathrm{MPa}$ is finally retained.

Figure 14 shows the adequation of the experimental and numerical curves, when using the parameters defined above.

\subsubsection{Validation and discussion}

Henceforth, a numerical computation is performed with $I=20 \mathrm{~mm}$ and the simulation response curve is first compared to the associated experimental ones in order to validate the 3-phase model as well as the choice of the cohesive properties in such extreme conditions. The numerical solution is plotted in Figure 15 together with two experimental curves corresponding to two different thicknesses (for comparison purposes, the force was divided by the current thickness). The general shape of the curves is similar from both experimental and numerical points of view. The only differences appear at the end of the test (just before failure), where the numerical model underestimates the ultimate resistance against cracking.

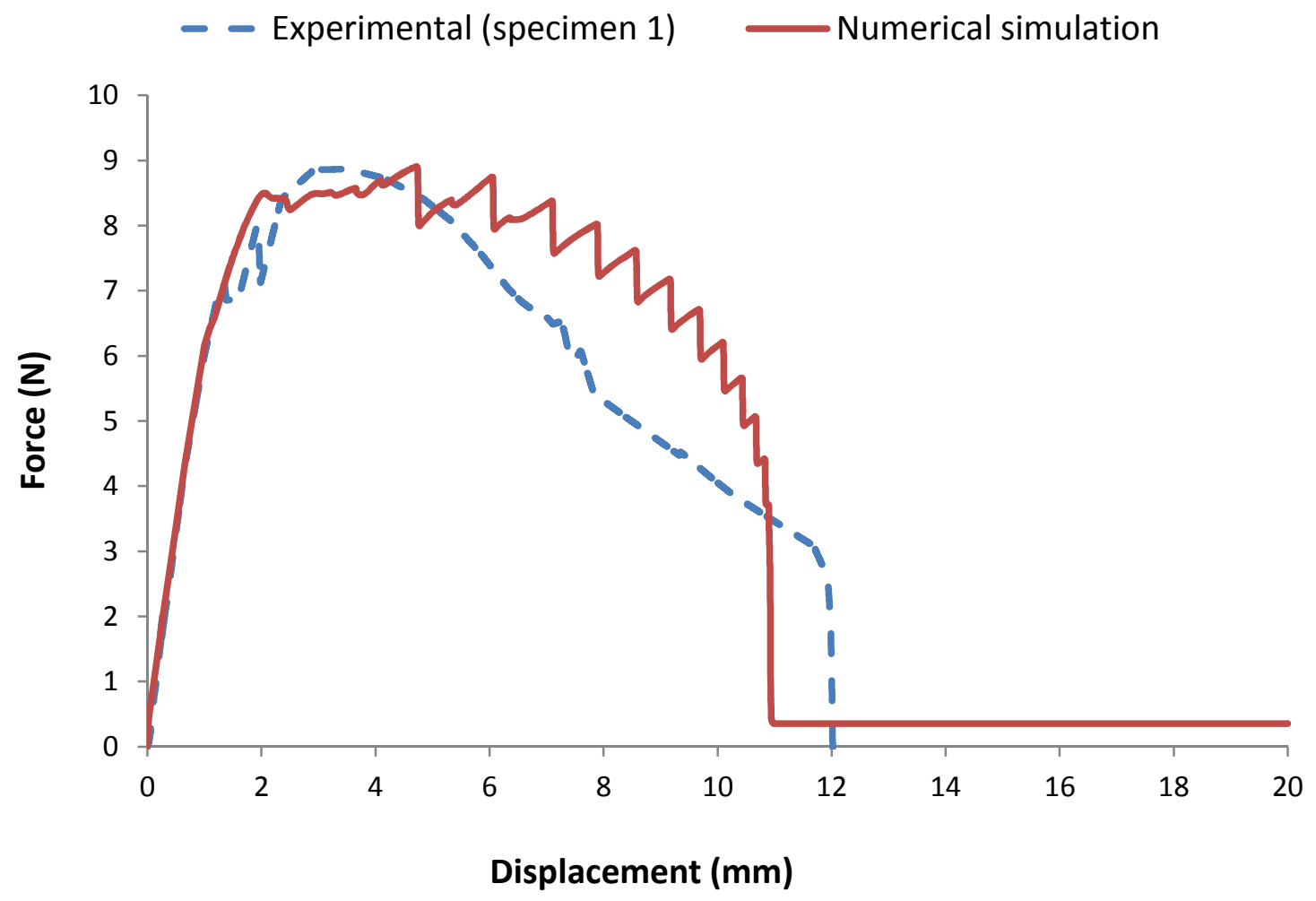

Fig. 14. Calibration of the cohesive parameters 


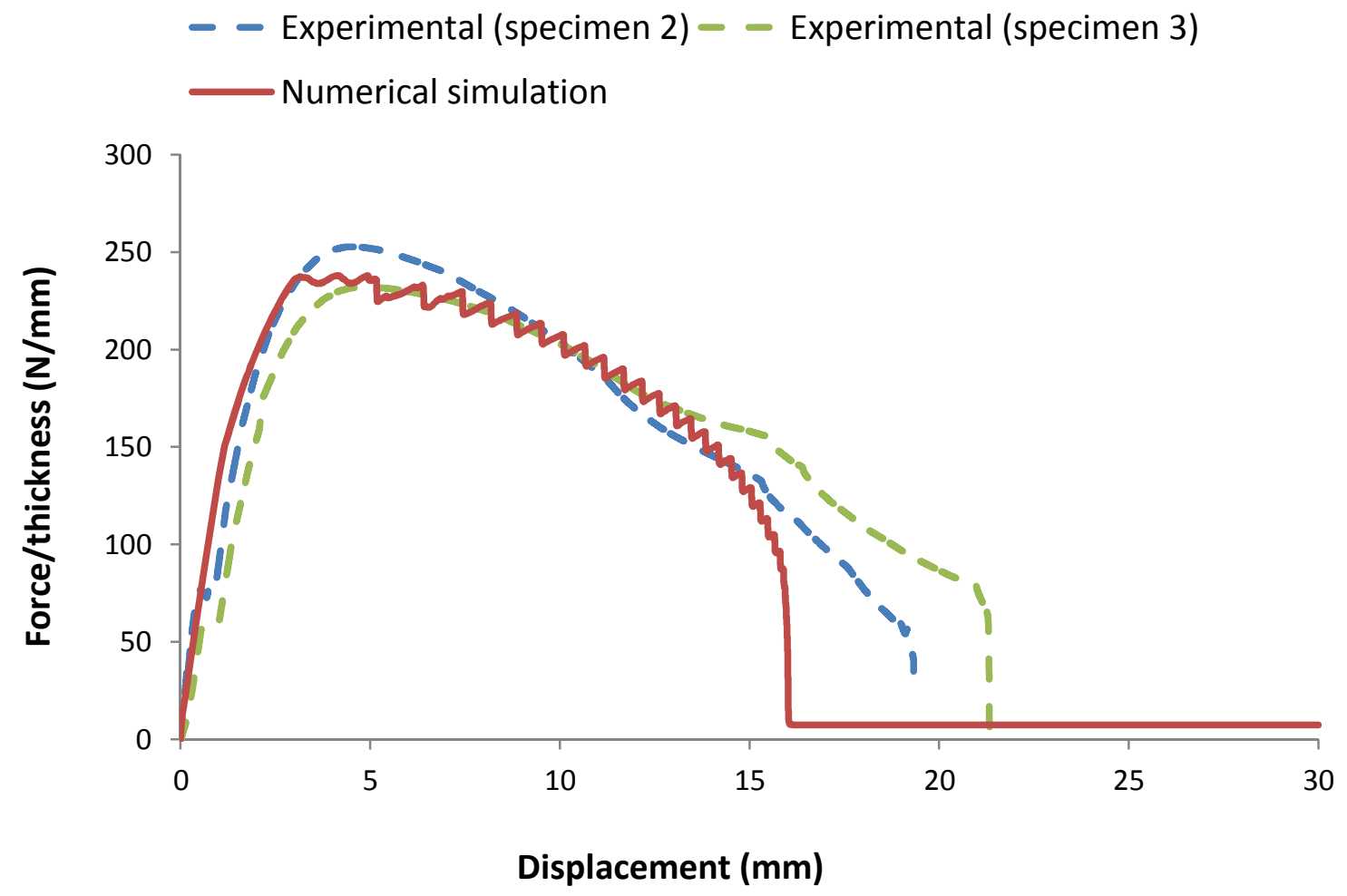

Fig. 15. Comparison between experimental and numerical results of DENT tests

Figure 16 depicts again Hencky strain distributions in the longitudinal direction at one particular loading level, obtained both experimentally (with the DIC method) and numerically (with the finite element model). Similar color scales have also been applied in both experimental and numerical graphs. From a qualitative point of view, the numerical and experimental deformed shapes are somewhat similar, but the crack lips remain particularly horizontal in the numerical simulation whereas they do not in practice. The numerical computation gives rise thus to longitudinal strains that are significantly concentrated in a small region (green zone) and rapidly drop down to very small strains (blue zone), while they decrease more gradually in reality as suggested by the broad spectrum of colors observed with the DIC method.

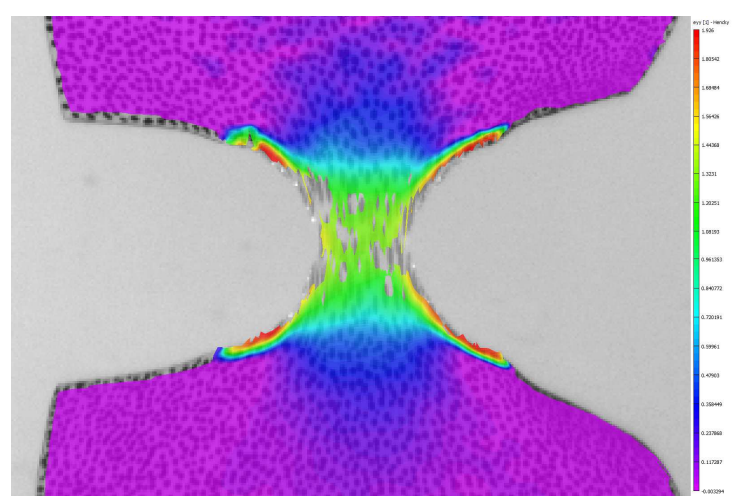

(DIC/maximum displacement $=15.57 \mathrm{~mm}$ )

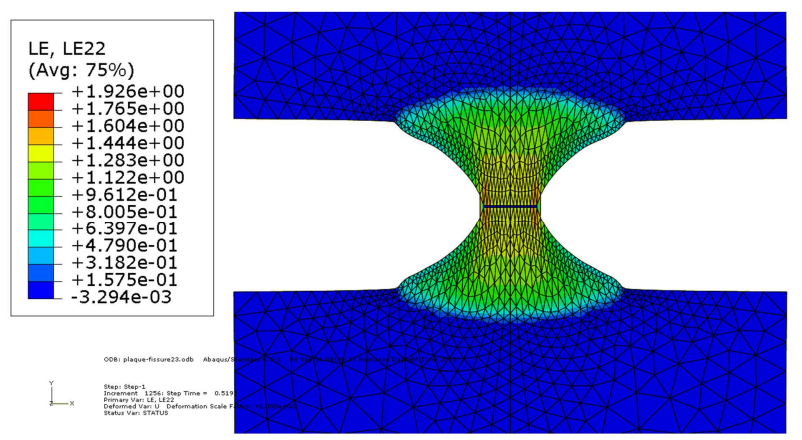

(FE/maximum displacement $=15.57 \mathrm{~mm})$

Fig. 16. Comparison between DIC and FE results of DENT specimens: longitudinal strain distributions for an arbitrary enforced displacement 


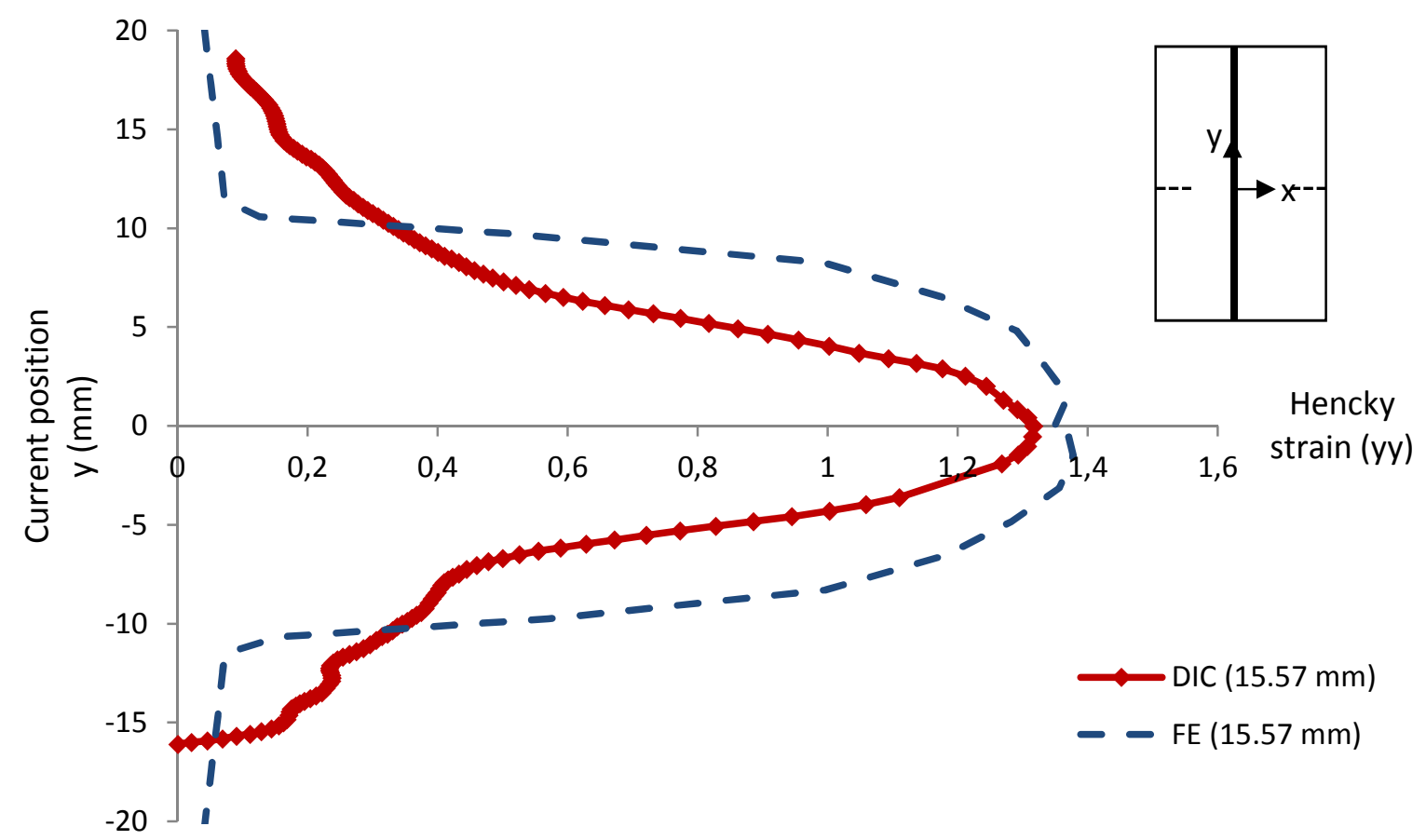

Fig. 17. Comparison between DIC and FE results of DENT specimens: longitudinal strain distributions for an arbitrary enforced displacement (along the vertical symmetry axis)

For a more quantitative comparison, the longitudinal Hencky strains have been plotted along the longitudinal symmetry axis, as seen in Figure 17. The strain profiles obtained both experimentally and numerically are in rather good accordance. The maximum longitudinal strain along this vertical path is observed at the center of the specimen and similar values are obtained by experimental and numerical approaches. However, the two curves displayed in Figure 17 confirm the fact that the strains derived from the numerical simulation are too much localized in the center of the specimen, near the crack tips. In order to overcome the difficulties related to the highly localized plastic deformations, one possible solution could be to develop a non-local model, incorporating the strain gradient effects, as recently performed by Voyiadjis et al. [38].

\section{Conclusions}

In this paper, the mechanical behavior of polyethylene films has been investigated in the framework of finite elastoplastic strains. An original 3-phase model, particularly devoted to semicrystalline polymers between their glass transition and melting temperatures, has been implemented in a finite element program. This constitutive model partly consists in two so-called "crystalline" and "amorphous" phases, represented by classical elastoplastic and hyperelastic behaviors, respectively. However, it differs from other models in that it contains a third transitional interphase, which is represented by a perfect plastic model with a new threshold stress based on a "multi-axial factor" as a function of the deformation state. The objective of the present paper is then to show the ability of this new model, and particularly of this intermediate phase, to explain the most significant features of the experimental behavior in several applications. 
Three series of experimental tests have thus been performed on unnotched, open-hole and Double Edge Notched Tensile rectangular specimens, respectively. The first tests, carried out on unnotched specimens in uniaxial tension, allow one to calibrate the material parameters to be used in subsequent finite element analyses. Then, the other problems are computed using a 2D finite element model in finite strains including the above constitutive model, and the experimental results are used for validation purposes, among other things.

In both cases of open-hole and DENT specimens, the experimental tests are conducted until failure of the specimens and the finite element computations are continued up to similar loading levels. Force-displacement curves are plotted first, in order to analyze the global response of the specimens. A good agreement is observed in both cases between experimental and simulation results, much better than when using a standard elastoplastic model calibrated on the same uniaxial curve. Furthermore, the experimental tests are post-processed with the help of 2D Digital Image Correlation. The results are compared to the strain distributions obtained through the numerical simulations. Again, the agreement is very satisfactory between the model results and the experimental ones.

The constitutive model used in this study has thus proved to be able to reproduce efficiently and accurately the biaxial behavior of semi-crystalline polymers in presence of stress concentrations or, better yet, of singularities due to the presence of cracks, up to very advanced deformation states.

\section{References}

[1] Huang, C.H., Wu, J.S., Huang, C.C., Lin, L.S., 2003. Adhesion, permeability and mechanical properties of multilayered blown films using maleated low-density polyethylene blends as adhesionpromoting layers. Polymer Journal 35 (12), 978-984.

[2] Kuhn, W., Grün, F., 1942. Beziehungen zwischen elastischen Konstanten und Dehnungsdoppelbrechung hochelastischer Stoffe. Kolloid-Zeitschrift 101 (3), 248-271.

[3] James, H.M., Guth, E., 1943. Theory of the elastic properties of rubber. Journal of Chemical Physics 11 (10), 455-481.

[4] Diani, J., Brieu, M., Vacherand. J.M., Rezgui, A., 2004. Directional model for isotropic and anisotropic hyperelastic rubber-like materials. Mechanics of Materials 36 (4), 313-321.

[5] Richeton, J., Ahzi, S., Vecchio, K.S., Jiang, F.C., Makradi, A., 2007. Modeling and validation of the large deformation inelastic response of amorphous polymers over a wide range of temperatures and strain rates. International Journal of Solids and Structures 44 (24), 7938-7954.

[6] van Dommelen, J.A.W., Parks, D.M., Boyce, M.C., Brekelmans, W.A.M., Baaijens, F.P.T., 2003. Micromechanical modeling of the elasto-viscoplastic behavior of semi-crystalline polymers. Journal of the Mechanics and Physics of Solids 51 (3), 519-541.

[7] Bergström, J.S., Kurtz, S.M., Rimnac, C.M., Edidin, A.A., 2002. Constitutive modeling of ultra-high molecular weight polyethylene under large-deformation and cyclic loading conditions. Biomaterials 23 (11), 2329-2343. 
[8] Lee, B.J., Argon, A.S., Parks, D.M., Ahzi, S., Bartczak, Z., 1993. Simulation of large strain plastic deformation and texture evolution in high density polyethylene. Polymer 34 (17), 3555-3575.

[9] Dupaix, R.B., Boyce, M.C., 2007. Constitutive modeling of the finite strain behavior of amorphous polymers in and above the glass transition. Mechanics of Materials 39 (1), 39-52.

[10] Richeton, J., Ahzi, S., Vecchio, K.S., Jiang, F.C., Adharapurapu, R.R., 2006. Influence of temperature and strain rate on the mechanical behavior of three amorphous polymers: Characterization and modeling of the compressive yield stress. International Journal of Solids and Structures 43 (7-8), 2318-2335.

[11] Gueguen, O., Richeton, J., Ahzi, S., Makradi, A., 2008. Micromechanically based formulation of the cooperative model for the yield behavior of semi-crystalline polymers. Acta Materialia 56 (7), 1650-1655.

[12] Ayoub, G., Zaïri, F., Fréderix, C., Gloaguen, J.M., Naït-Abdelaziz, M., Seguela, R., Lefebvre, J.M., 2011. Effects of crystal content on the mechanical behaviour of polyethylene under finite strains: Experiments and constitutive modelling. International Journal of Plasticity 27 (4), 492-511.

[13] Ponçot, M., Addiego, F., Dahoun, A., 2013. True intrinsic mechanical behaviour of semicrystalline and amorphous polymers: Influences of volume deformation and cavities shape. International Journal of Plasticity 40, 126-139.

[14] Regrain, C., Laiarinandrasana, L., Toillon, S., Saï, K., 2009. Multi-mechanism models for semicrystalline polymer: Constitutive relations and finite element implementation. International Journal of Plasticity 25 (7), 1253-1279.

[15] Khan, F., Yeakle, C., Gomaa, S., 2012. Characterization of the mechanical properties of a new grade of ultra high molecular weight polyethylene and modeling with the viscoplasticity based on overstress. Journal of the Mechanical Behavior of Biomedical Materials 6, 174-180.

[16] Farrokh, B., Khan, A.S., 2010. A strain rate dependent yield criterion for isotropic polymers: Low to high rates of loading. European Journal of Mechanics - A/Solids 29 (2), 274-282.

[17] Rozanski, A., Galeski, A., 2013. Plastic yielding of semicrystalline polymers affected by amorphous phase. International Journal of Plasticity 41, 14-29.

[18] Hachour, K., Zaïri, F., Naït-Abdelaziz, M., Gloaguen, J.M., Aberkane, M., Lefebvre, J.M., 2014. Experiments and modeling of high-crystalline polyethylene yielding under different stress states. International Journal of Plasticity 54, 1-18.

[19] Srivastava, V., Chester, S.A., Ames, N.M., Anand, L., 2010. A thermo-mechanically-coupled largedeformation theory for amorphous polymers in a temperature range which spans their glass transition. International Journal of Plasticity 26 (8), 1138-1182.

[20] Uchida, M., Tada, N., 2013. Micro-, meso- to macroscopic modeling of deformation behavior of semi-crystalline polymer. International Journal of Plasticity 49, 164-184. 
[21] Maurel-Pantel, A., Baquet, E., Bikard, J., Bouvard, J.L., Billon, N., 2015. A thermo-mechanical large deformation constitutive model for polymers based on material network description: Application to a semi-crystalline polyamide 66. International Journal of Plasticity 67, 102-126.

[22] Zeng, F., Le Grognec, P., Lacrampe, M.F., Krawczak, P., 2010. A constitutive model for semicrystalline polymers at high temperature and finite plastic strain: Application to PA6 and PE biaxial stretching. Mechanics of Materials 42 (7), 686-697.

[23] Kajberg, J., Sundin, K.G., Melin, L.G., Ståhle, P., 2004. High strain-rate tensile testing and viscoplastic parameter identification using microscopic high-speed photography. International Journal of Plasticity 20 (4-5), 561-575.

[24] Yoshida, F., 2000. A constitutive model of cyclic plasticity. International Journal of Plasticity 16 (3-4), 359-380.

[25] Besnard, G., Hild, F., Roux, S., 2006. "Finite-element" displacement fields analysis from digital images: Application to Portevin-Le Châtelier bands. Experimental Mechanics 46 (6), 789-803.

[26] Avril, S., Pierron, F., Sutton, M.A., Yan, J., 2008. Identification of elasto-visco-plastic parameters and characterization of Lüders behavior using digital image correlation and the virtual fields method. Mechanics of Materials 40 (9), 729-742.

[27] Sutton, M.A., Walters, W.J., Peters, W.H., Ranson, W.F., McNeill, S.R., 1983. Determination of displacements using an improved digital correlation method. Image and Vision Computing 1 (3), 133139.

[28] Sutton, M.A., Cheng, M., Peters, W.H., Chao, Y.J., McNeill, S.R., 1986. Application of an optimized digital correlation method to planar deformation analysis. Image and Vision Computing 4 (3), 143150.

[29] Balieu, R., Lauro, F., Bennani, B., Haugou, G., Chaari, F., Matsumoto, T., Mottola, E., 2015. Damage at high strain rates in semi-crystalline polymers. International Journal of Impact Engineering $76,1-8$.

[30] Uchida, M., Tada, N., 2011. Sequential evaluation of continuous deformation field of semicrystalline polymers during tensile deformation accompanied by neck propagation. International Journal of Plasticity 27 (12), 2085-2102.

[31] Hong, K., Rastogi, A., Strobl, G., 2004. A model treating tensile deformation of semi-crystalline polymers: Quasi-static stress-strain relationship and viscous stress determined for a sample of polyethylene. Macromolecules 37 (26), 10165-10173.

[32] Drozdov, A.D., Christiansen, J.deC., 2008. Thermo-viscoelastic and viscoplastic behavior of highdensity polyethylene. International Journal of Solids and Structures 45 (14-15), 4274-4288.

[33] Peacock, A.J., 2000. Handbook of Polyethylene: Structures, Properties and Applications. Marcel Dekker.

[34] Arruda, E.M., Boyce, M.C., 1993. A three-dimensional constitutive model for the large stretch behavior of rubber elastic materials. Journal of the Mechanics and Physics of Solids 41 (2), 389-412. 
[35] Baghani, M., Arghavani, J., Naghdabadi, R., 2014. A finite deformation constitutive model for shape memory polymers based on Hencky strain. Mechanics of Materials 73, 1-10.

[36] Bergström, J.S., Boyce, M.C., 1998. Constitutive modeling of the large strain time-dependent behavior of elastomers. Journal of the Mechanics and Physics of Solids 46 (5), 931-954.

[37] Zeng, F., 2010. On the modeling of the mechanical behavior in finite elastoplasticity of shrink wrap films (Contribution à la modélisation du comportement mécanique en grandes déformations élastoplastiques de films plastiques d'emballage). Ph.D. thesis, University of Lille, France.

[38] Voyiadjis, G.Z., Shojaei, A., Mozaffari, N., 2014. Strain gradient plasticity for amorphous and crystalline polymers with application to micro- and nano-scale deformation analysis. Polymer 55 (16), 4182-4198. 